\title{
A stochastic-statistical residential burglary model with independent Poisson clocks
}

\author{
CHUNTIAN WANG ${ }^{1}$, YUAN ZHANG ${ }^{2}$, ANDREA L. BERTOZZI $^{3}$ \\ and MARTIN B. SHORT \\ ${ }^{1}$ Department of Mathematics, The University of Alabama, Tuscaloosa, AL 35487, USA \\ email: cwang27@ua.edu \\ ${ }^{2}$ School of Mathematical Sciences, Peking University, Beijing, China, 100871 \\ email: zhangyuan@math.pku.edu.cn \\ ${ }^{3}$ Departments of Mathematics and Mechanical and Aerospace Engineering \\ University of California, Los Angeles, Los Angeles, CA 90095, USA \\ email: bertozzi@ucla.edu \\ ${ }^{4}$ School of Mathematics, Georgia Institute of Technology, Atlanta, GA 30332, USA \\ email:mbshort@math.gatech.edu
}

(Received 12 August 2019; revised 31 December 2019; accepted 7 January 2020; first published online 5 February 2020)

Residential burglary is a social problem in every major urban area. As such, progress has been to develop quantitative, informative and applicable models for this type of crime: (1) the Deterministictime-step (DTS) model [Short, D’Orsogna, Pasour, Tita, Brantingham, Bertozzi \& Chayes (2008) Math. Models Methods Appl. Sci. 18, 1249-1267], a pioneering agent-based statistical model of residential burglary criminal behaviour, with deterministic time steps assumed for arrivals of events in which the residential burglary aggregate pattern formation is quantitatively studied for the first time; (2) the SSRB model (agent-based stochastic-statistical model of residential burglary crime) [Wang, Zhang, Bertozzi \& Short (2019) Active Particles, Vol. 2, Springer Nature Switzerland AG, in press], in which the stochastic component of the model is theoretically analysed by introduction of a Poisson clock with time steps turned into exponentially distributed random variables. To incorporate independence of agents, in this work, five types of Poisson clocks are taken into consideration. Poisson clocks (I), (II) and (III) govern independent agent actions of burglary behaviour, and Poisson clocks (IV) and (V) govern interactions of agents with the environment. All the Poisson clocks are independent. The time increments are independently exponentially distributed, which are more suitable to model individual actions of agents. Applying the method of merging and splitting of Poisson processes, the independent Poisson clocks can be treated as one, making the analysis and simulation similar to the SSRB model. A Martingale formula is derived, which consists of a deterministic and a stochastic component. A scaling property of the Martingale formulation with varying burglar population is found, which provides a theory to the finite size effects. The theory is supported by quantitative numerical simulations using the pattern-formation quantifying statistics. Results presented here will be transformative for both elements of application and analysis of agent-based models for residential burglary or in other domains.

Key words: Applications of continuous-time Markov processes on discrete state spaces, applications of stochastic analysis (to partial differential equations (PDEs), etc.), PDEs in connection with game theory, economics, social and behavioural sciences

2010 Mathematics Subject Classification: 60G51 (Primary); 35Q91 (Secondary) 


\section{Introduction}

Since residential burglary is a critical social issue in every major urban area, quantitative, informative and applicable crime models are called for inside and outside of the scientific community. Mathematical modelling and prediction of crime have been a burgeoning area for more than 10 years and many works have been done (e.g., [3, 4, 6, 11, 12, 38, 39, 56, 59, 60, 66, 70, 72, 76, $77,78,81,84,86,87,88,90,91,92,93,94,98,99,104,106,108])$.

One prominent feature of urban residential burglary is that the crimes are not distributed uniformly in time and space but rather exhibit spatio-temporal aggregates called 'hotspots'. In [93], a pioneering agent-based statistical model of criminal behaviour for residential burglary (referred to as the DTS model) is developed and the aggregate pattern formation is quantitatively studied for the first time. Interactions of agent actions with the environment are described based on the near-repeat victimisation and the broken-windows effects, which are notions in criminology and sociology that have been empirically observed [10, 32, 43]. Follow-up works of [93] show that such simple statistical agent-based model can exhibit both crime displacement and crime suppression in the presence of police activity $[90,91]$. In the DTS model, time steps are discretised with a fixed duration and occur at regular intervals, such that all types of events for all agents arrive according to the same schedule. However, a more realistic model should treat all events as occurring independently, according to their own stochastic clock.

To incorporate randomness of arrivals of events into the DTS model, in [104], a Poisson clock is applied to the DTS model [93] and the time steps are turned into exponentially distributed random variables, and further stochasticity is brought into the DTS model. The model in [104] is referred to as the SSRB model (agent-based stochastic-statistical model of residential burglary crime). The introduction of a Poisson clock into the residential burglary model brings in theoretically the mathematical framework of Markov pure jump processes and interacting particle systems ([20, 62, 63, 64, 65]). A Martingale formulation is derived for the SSRB model to express the model as the summation of two components: a deterministic component and a stochastic component. Together with statistics quantifying the degree of hotspot transience, analysis of the stochastic component leads to a theory for the finite size effects present in the system. These effects are observed in the simulations of the DTS model and the SSRB model: both transient and stationary hotspot dynamical regimes appear in the simulations, and as burglar number decreases, simulations exhibit more transience. Since the number of operating criminals within any city is very likely to fall within the regime of hotspot transience observed, a deeper and quantitative understanding of the finite size effects is relevant to real crime statistics $[38,76]$. This is the first time that stochastic analysis is applied into residential burglary models and the stochastic component is theoretically analysed.

Despite the progress made by the SSRB model, it still has some limitations. Most notably, in the SSRB model, only one Poisson clock governs all the agents, with all agents acting simultaneously whenever the clock randomly ticks. However, in reality, criminals (or very small groups of criminals acting as teams) act independently. Therefore, models are called for in which independent actions of agents are taken into account. In fact, within the context of the DTS family of models, five types of independent Poisson clocks need to be taken into consideration to describe each agent's burglary behaviour and their interactions with the environment as well. 
Poisson clocks (I) are assigned to individual agents to govern their burgling, Poisson clocks (II) govern their movement, Poisson clocks (III) govern replacement of agents, and Poisson clocks (IV) and Poisson clocks (V) govern the implementation of the near-repeat victimisation and the broken-windows effects. All these Poisson clocks should be independent.

In this work, we introduce Poisson clocks (I), (II), (III), (IV) and (V) into the SSRB model, so that agents will act individually instead of acting simultaneously. This residential burglary model will be referred to as the SSRB-IPC model (agent-based stochastic-statistical model of residential burglary with independent Poisson clocks). The methodology of the theoretical analysis and numerical simulations of the SSRB-IPC model employs theory regarding the merging and splitting of Poisson processes [7], and the independent Poisson processes associated with the independent Poisson clocks can be treated as one merged Poisson process wherein the Poisson clocks compete to advance first in the merged Poisson process with probability in proportion to the rate of each Poisson clock. This method allows the analysis and computation of the SSRBIPC model to be very similar to that of the SSRB model [104], and the simulation cost of the SSRB-IPC model is reduced.

A Martingale formula of the SSRB-IPC model is derived, which consists of a deterministic and a stochastic component. The deterministic component leads to a hydrodynamic-limit-type continuum analogue of the SSRB-IPC model. The continuum analogue of the SSRB-IPC model coincides with that of the DTS model [93] and of the SSRB model [104]. The stochastic component of the SSRB-IPC model is less complicated to compute and analyse than that of the SSRB model, as all the covariances vanish, thanks to the independence of the Poisson clocks. A scaling property of the stochastic component with varying burglar population is found that provides a theory to the finite size effects. The theory is supported by quantitative numerical simulations using the statistics quantifying the degree of hotspot transience first developed in Ref. [104].

The theoretical methods and results found here have broad applicability. Poisson processes are widely applied in chemistry, biology, physics, etc. (see, e.g., [2, 5, 18, 25, 37, 80]). However, as far as we know there are not many applications of independent Poisson clocks into modelling in social science. Many existing social science models do not address independence of arrivals of events explicitly [19, 61, 67, 89, 107]. Stochastic models are increasingly applied as a tool to social sciences, e.g., urban structure, disease transmission and networks $([24,33,34])$. What is more, pattern formation arises in many complex systems such as social science $([14,35,41,42,45,48,57,58,82,99,100,105])$. But quantitative study has been lacking so far. The mathematical framework with the application of stochastic analysis and quantification of pattern formation developed here may be therefore broadly useful.

The article is organised as follows. In Section 2, the SSRB-IPC model is introduced and numerical simulations of the model explored finite size effects. The Martingale formulation is derived in Section 3.1. Based on the Martingale formulation, a continuum analogue of the SSRB-IPC model is derived (Section 3.2). The degree of hotspot transience of the simulations of the SSRB-IPC model and its continuum analogue is quantitatively measured using statistics (Section 3.3). In Section 3.4, the finite size effects are analysed theoretically based upon the Martingale formulation. The theory is supported by quantitative simulations. The conclusion is in Section 4. 


\section{The SSRB-IPC model and simulations}

\subsection{The SSRB-IPC model}

The agent-based SSRB-IPC model consists of essentially two components - the stationary burglary sites and burglar agents jumping from site to site, occasionally committing crimes. Independent Poisson clocks will govern time increments of evolution of these components.

We assume the domain as $\mathscr{D}:=[0, L] \times[0, L]$ with periodic boundary conditions. The lattice grid over the domain $\mathscr{D}$ has spacing $\ell=L / N, N \in \mathbb{N}$. The grid points are denoted as $\mathbf{s}=\left(\mathbf{s}_{1}, \mathbf{s}_{2}\right)$, $\mathbf{s}_{1}=\ell, 2 \ell, \cdots, L, \mathbf{s}_{2}=\ell, 2 \ell, \cdots, L$. And the collection of all the grid points is denoted as $\mathscr{S}^{\ell}$. Attached to each grid point $\mathbf{s} \in \mathscr{S}^{\ell}$ is a pair $\left(n_{\mathbf{s}}^{\ell}(t), A_{\mathbf{s}}^{\ell}(t)\right)$ representing the number of burglar agents and attractiveness at site $\mathbf{s}$ at time $t$. The attractiveness shows the burglar's beliefs about the vulnerability and value of the site. We also assume that $A_{\mathbf{s}}^{\ell}(t)$ consists of a dynamic term and a static background term

$$
A_{\mathbf{s}}^{\ell}(t)=B_{\mathbf{s}}^{\ell}(t)+A 0_{\mathbf{s}}^{\ell}
$$

Here $A 0_{\mathbf{s}}^{\ell}$ is not necessarily uniform over the lattice grids. And the initial data are given to be $\left(n_{\mathbf{s}}^{\ell}(0), B_{\mathbf{s}}^{\ell}(0)\right)=\left(n 0_{\mathbf{s}}^{\ell}, B 0_{\mathbf{s}}^{\ell}\right)$.

A type (I) Poisson clock is assigned to each agent to govern his action of burgling. Suppose that a type (I) clock of an agent advances at time $t^{-}$. At time $t$, the agent will burgle his current location and immediately be removed from the system, representing the burglar fleeing with his trophy. Type (I) Poisson clocks advance according to independent Poisson processes with rate $A_{\mathbf{s}}^{\ell}(t)$, $\mathbf{s}$ being the current location of the burglar agent. Thus, the attractiveness numerically represents the stochastic rate of burglary at a location, given that there is a criminal located there.

A type (II) Poisson clock is assigned to each agent to govern his movement. Suppose that a type (II) clock of an agent advances at time $t^{-}$. At time $t$, the agent will jump from site $\mathbf{s}$ to one of the neighbouring sites, say $\mathbf{k}$, with a probability, $q_{\mathbf{s} \rightarrow \mathbf{k}}^{\ell}(t)$, which is defined by the ratio of the attractiveness of $\mathbf{k}$ over the combined attractiveness of the neighbouring sites of $\mathbf{s}$ :

$$
q_{\mathbf{s} \rightarrow \mathbf{k}}^{\ell}(t)=\frac{A_{\mathbf{k}}^{\ell}\left(t^{-}\right)}{T_{\mathbf{s}}^{\ell}\left(t^{-}\right)},
$$

where $T_{\mathbf{s}}^{\ell}(t):=\sum_{\substack{\mathbf{s}^{\prime} \sim \mathbf{s} \\ \mathbf{s}^{\prime}}} A_{\mathbf{s}^{\prime}}^{\ell}(t), \mathbf{s}^{\prime} \sim \mathbf{s}$ indicating all of the neighbouring sites of $\mathbf{s}$. To create consistency with the DTS model, type (II) Poisson clocks advance according to independent Poisson processes with rate $D \ell^{-2}$, where $D$ is an absolute constant independent of $\ell$. On average, the time increment $\delta t$ for type (II) clocks is the inverse of the rate of the Poisson clock:

$$
\delta t \cong \frac{\ell^{2}}{D}
$$

A type (III) Poisson clock is assigned to each site to govern replacement of burglars. Suppose that the type (III) clock attached to a site $\mathbf{s}$ advances at time $t^{-}$. At time $t$, a new agent will be placed at s. Type (III) Poisson clocks advance according to independent Poisson processes with rate $\Gamma$, an absolute constant indicating the growth rate of criminal population. 
The attractiveness field gets updated according to the repeat and near-repeat victimisation and the broken-windows effect. The 'broken windows' theory argues that the visible signs of past crimes are likely to create an environment that encourages further illegal activities [106]. The so-called repeat and near-repeat events depict the phenomenon that residential burglars prefer to return to a previously burgled house and its neighbours [30, 49, 50, 51, 92].

The repeat victimisation and broken-windows effects are modelled by letting $B_{\mathbf{s}}^{\ell}$ depend upon previous burglary events at site s. Let $\theta$ be an absolute constant measuring strength of the repeat victimisation effect, and the attractiveness increases by $\theta$ whenever a burglary event occurs on that site via a type (I) clock as described above. However, this increase has a finite lifetime governed by type (IV) Poisson clocks, one of which is assigned to each site. Suppose that the type (IV) clock associated with site $\mathbf{s}$ advances at time $t^{-}$. At time $t$, the attractiveness gets updated as follows:

$$
B_{\mathbf{s}}^{\ell}(t)=B_{\mathbf{s}}^{\ell}\left(t^{-}\right)\left(1-\frac{\omega}{D \ell^{\sigma}}\right)
$$

where $\omega$ is an absolute constant setting the speed of the decay. Type (IV) Poisson clocks advance according to independent Poisson processes with rate $D \ell^{\sigma}$, where $\sigma<0$ is an absolute constant that affects the speed of this type (IV) process, and whose value we will decide later. On average, the time increment $\delta t$ for the type (IV) clocks is the inverse of the Poisson clock rate

$$
\delta t \cong \frac{1}{D \ell^{\sigma}}
$$

The near-repeat victimisation effect is modelled by allowing $B_{\mathbf{s}}^{\ell}$ to spread in space from each house to its neighbours via a type (V) Poisson clock, which is assigned to each site. Suppose that the type (V) clock associated with a site $\mathbf{s}$ advances at time $t^{-}$. At time $t$, the attractiveness gets updated as follows:

$$
B_{\mathbf{s}}^{\ell}(t)=B_{\mathbf{s}}^{\ell}\left(t^{-}\right)+\frac{\eta}{4} \ell^{2} \Delta^{\ell} B_{\mathbf{s}^{\prime}}^{\ell}\left(t^{-}\right),
$$

where $\eta \in(0,1)$ is an absolute constant that measures the significance of neighbourhood effects, and $\Delta^{\ell}$ is the discrete spatial Laplace operator associated with the lattice grid, namely

$$
\Delta^{\ell} B_{\mathbf{s}}^{\ell}(t)=\ell^{-2}\left(\sum_{\substack{\mathbf{s}^{\prime} \\ \mathbf{s}^{\prime} \sim \mathbf{s}}} B_{\mathbf{s}^{\prime}}^{\ell}(t)-4 B_{\mathbf{s}}^{\ell}(t)\right)
$$

Again for consistency with the DTS model, type (V) Poisson clocks advance according to independent Poisson processes with rate $D \ell^{-2}$.

The spatially homogeneous equilibrium solutions to the above described SSRB-IPC model are the same as the DTS model [93] and the SSRB model [104]. From now on, we always assume that $A 0_{\mathrm{s}}^{\ell} \equiv A 0$ for simplicity. The homogeneous equilibrium values are deduced as 


$$
\bar{B}=\frac{\theta \Gamma}{\omega}, \quad \bar{n}^{\ell}=\frac{\Gamma \ell^{2}}{D\left(1-e^{-\frac{\ell^{2} \bar{A}}{D}}\right)} \cong \frac{\Gamma}{\bar{A}},
$$

where $\bar{A}=\bar{B}+A 0$.

\subsection{Numerical simulations of the SSRB-IPC model}

To compare the agent-based SSRB-IPC model with the agent-based SSRB model [104], we perform simulations of the models and display the resulting attractiveness fields in Figures 1, 2, 3 and 4. The SSRB model is shown in Figures 1(a), (b), 2(a), (b), 3(a), (b), 4(a) and (b). The SSRB-IPC model is shown in the remaining figures. The parameters are mostly the equivalent of those used to create the plots for the DTS model [93] in Figure 3 of [93] and for the SSRB model in Figures 2, 3 and 4 of [104]. The same behavioural regimes are observed:

(1) Spatial homogeneity. In this regime, $A_{\mathbf{s}}^{\ell}(t)$ does not vary essentially in space or in time. Very few visible hotspots, that is, the accumulation of $A_{\mathbf{s}}^{\ell}(t)$ in space and in time, appear in the process.

(2) Dynamic hotspots. In this regime, localised spots of increased attractiveness form and are transient. These spots remain for varying lengths of time and may appear and disappear at seemingly random locations and move about in space and deform over time. They may also merge and split. Similar examples of transitory hotspots have been observed in the simulation output in [93] and [104].

(3) Stationary hotspots. In this regime, hotspots form and stay more or less stationary over time.

The spatially homogeneous equilibrium value of the dynamic attractiveness $\bar{B}$ in (2.7) serves as a midpoint for all the simulations and is shaded in green. To document the false colour map for the attractiveness, a colour key is given in the figures. All the simulations were run with $L=128$, $\ell=1, \omega=1 / 15, D=100$, and $A 0=1 / 30$. As $\ell=1$, there is no need to specify $\sigma$ for type (IV) Poisson clocks. And the initial criminal number at each site $n 0_{\mathbf{s}}^{\ell}$ is set to be $\bar{n}^{\ell}$ on average, and the burglar agents are assumed to be randomly uniformly distributed over the $128 \times 128$ grids, with $\sum_{\mathbf{s} \in \mathscr{S}_{\ell}} n 0_{\mathbf{s}}^{\ell}=128^{2} \bar{n}^{\ell}$.

In all panels of Figures 1, 2, 3 and 4, we set $\Gamma=0.0019 q, \theta=5.6 / q$, where $q$ takes on values 1 , $\sqrt{10}, 10$ and $10 \sqrt{10}$ in panels 1 (a), 1(b), 2(a) and 2(b), respectively. This same pattern of $q$ values also applies to the panel sets 3(a), 3(b), 4(a) and 4(b); 1(c), 1(d), 2(c) and 2(d); and 3(c), 3(d), 4(c) and 4(d). For both SSRB and SSRB-IPC models, as $q$ increases, (2.7) implies that the initial burglar population and the burglar replacement rate both increase while the initial attractiveness field remains fixed.

For the cases with zero hotspot formation (Figures 1(a), (b), (c) and (d) and 2(a), (b), (c) and (d)), we set $\eta=0.2$ and $B 0_{\mathbf{s}}^{\ell} \equiv \bar{B}$ for every $\mathbf{s} \in \mathscr{S}^{\ell}$. For the cases with hotspot formation (the remaining figures), $\eta=0.03$, and $B 0_{\mathrm{s}}^{\ell}$ is set to be $\bar{B}$ on every site except for 30 randomly chosen grid points where the attractiveness is increased by 0.002 .

The same finite size effects as in the SSRB model [104] and the DTS model [93] are observed. The degree of hotspot 'transience' seems to depend on the total burglar population. The regimes of transient hotspots seem to appear associated with low or vanishing burglar numbers and low numbers of events, while the regimes of stationarity, including the stationary hotspots or homogeneity regimes, occur more likely with large numbers of burglars and burglary events. 

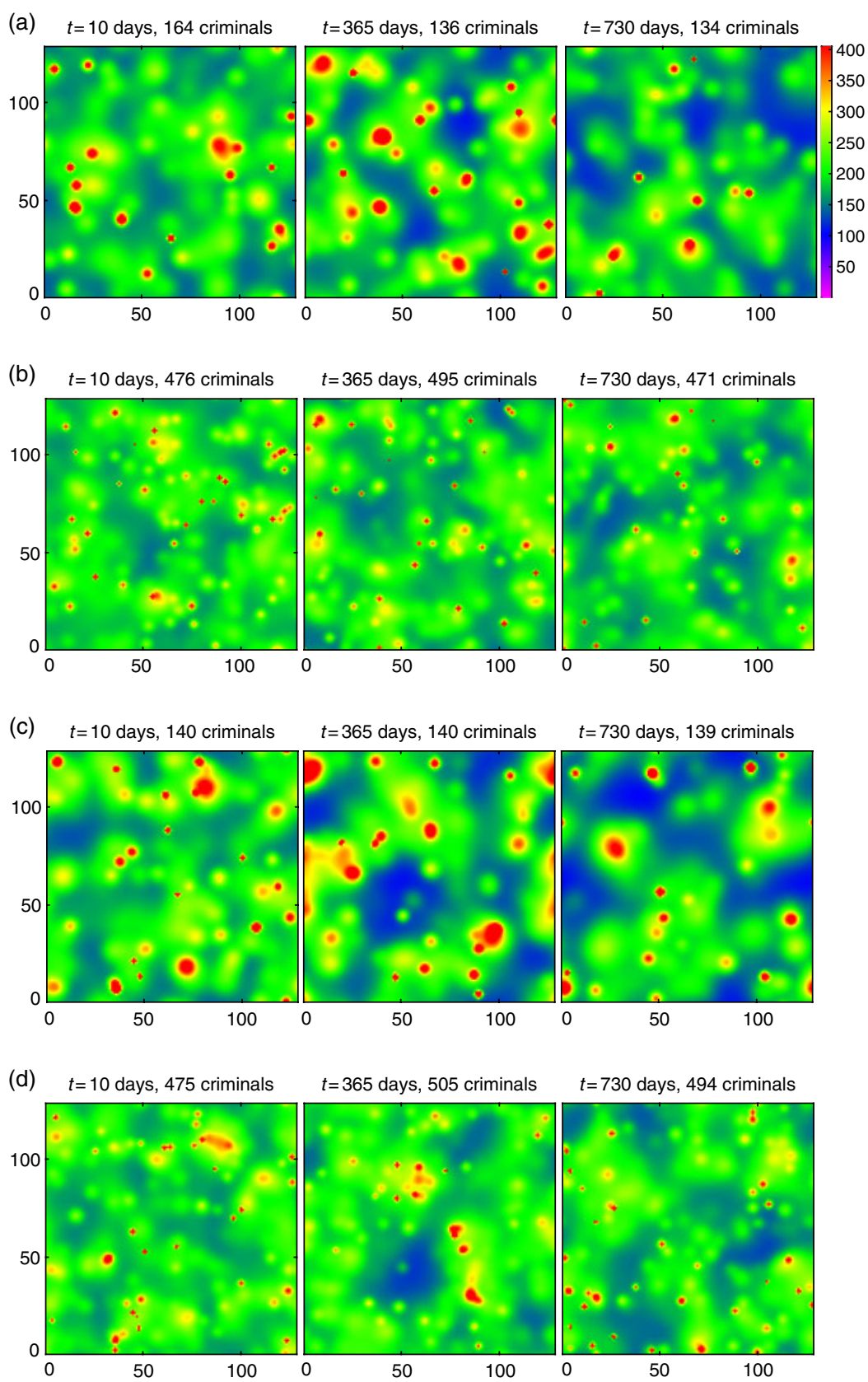

FIGURE 1. Plot of the attractiveness $A_{\mathbf{s}}^{\ell}(t)$ for the SSRB model [104] and the SSRB-IPC model. For both models, the initial conditions (at $t=0$ ) and parameters are set as $A 0=1 / 30, B 0_{\mathrm{s}}^{\ell} \equiv \bar{B}, n 0_{\mathrm{s}}^{\ell} \cong \bar{n}^{\ell}, D=100$, $L=128, \ell=1, \omega=1 / 15$, and $\eta=0.2$. For the SSRB model [104], in (a) and (b), we set $\Gamma=0.0019 q$, $\theta=5.6 / q, q=1$ in (a), and $q=\sqrt{10}$ in (b). For the SSRB-IPC model, in (c) and (d), we set $\Gamma=0.0019 q$, $\theta=5.6 / q, q=1$ in (c), and $q=\sqrt{10}$ in (d). (a) and (c) show the dynamic hotspot regimes, while (b) and (d) show less degree of transience of hotspots. 

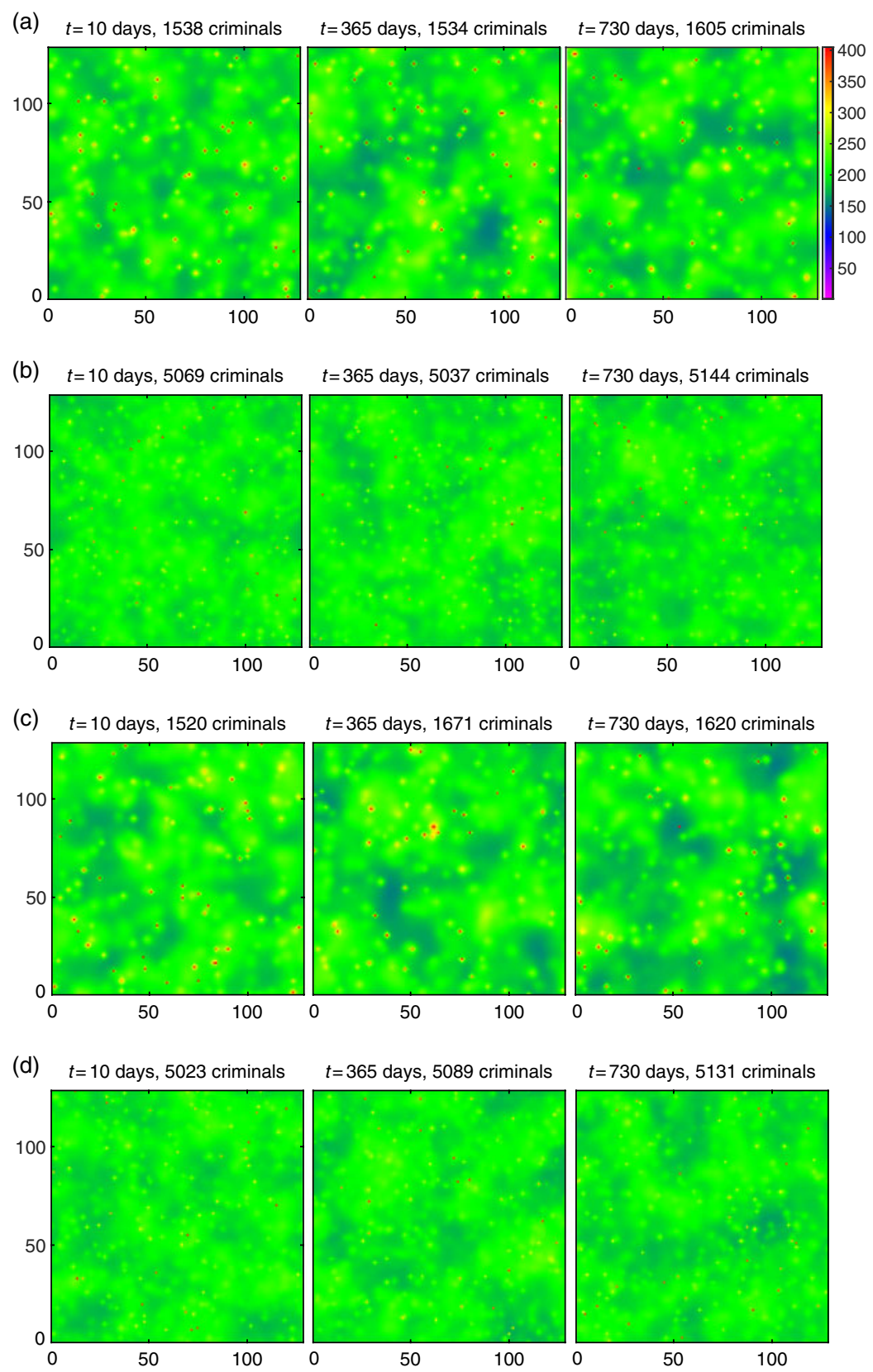

FIGURE 2. Plot of the attractiveness $A_{\mathrm{s}}^{\ell}(t)$ for the SSRB model [104] and the SSRB-IPC model. For both models, the initial conditions (at $t=0$ ) and parameters are set as $A 0=1 / 30, B 0_{\mathrm{s}}^{\ell} \equiv \bar{B}, n 0_{\mathrm{s}}^{\ell} \cong \bar{n}^{\ell}, D=100$, $L=128, \ell=1, \omega=1 / 15$ and $\eta=0.2$. For the SSRB model [104], in (a) and (b), we set $\Gamma=0.0019 q$, $\theta=5.6 / q, q=10$ in (a), and $q=10 \sqrt{10}$ in (b). For the SSRB-IPC model, in (c) and (d), we set $\Gamma=0.0019 q$, $\theta=5.6 / q, q=10$ in (c), and $q=10 \sqrt{10}$ in (d). (a) and (c) show the spatially homogeneous regimes, and (b) and (d) show even less transience of hotspots. 

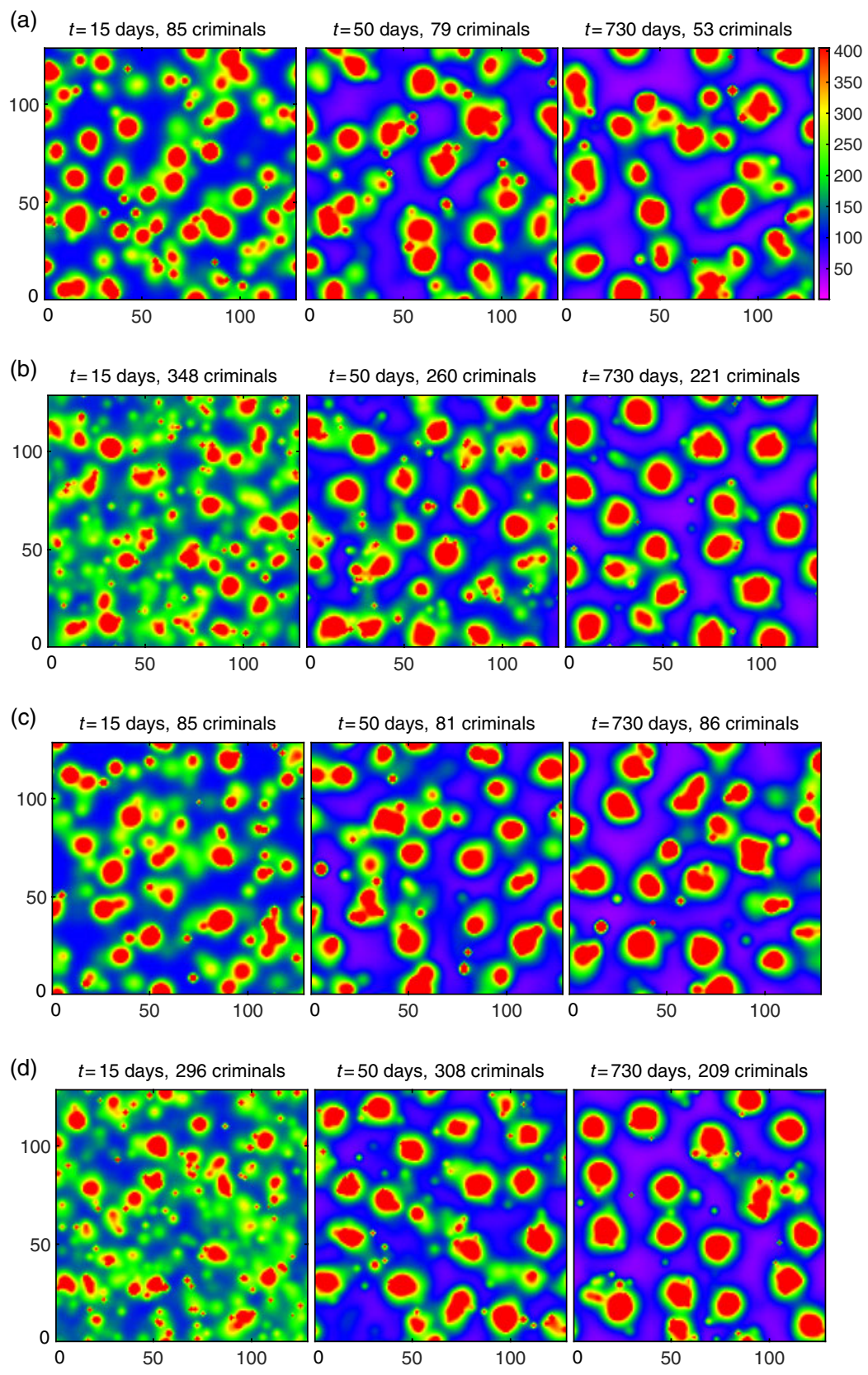

FIGURE 3. Plot of the attractiveness $A_{\mathbf{s}}^{\ell}(t)$ for the SSRB model [104] and the SSRB-IPC model. For both models, the parameters and initial conditions (at $t=0$ ) are set as $L=128, \ell=1, D=100, \omega=1 / 15, \eta=$ $0.03, A 0=1 / 30, n 0_{\mathrm{s}}^{\ell} \cong \bar{n}^{\ell}$, and $B 0^{\ell}$ is set to be $\bar{B}$ except for sites with slight perturbations. For the SSRB model [104], in (a) and (b), we set $\Gamma=0.0019 q, \theta=5.6 / q, q=1$ in (a), and $q=\sqrt{10}$ in (b). For the SSRBIPC model, in (c) and (d), we set $\Gamma=0.0019 q, \theta=5.6 / q, q=1$ in (c), and $q=\sqrt{10}$ in (d). (a) and (c) show the the dynamic hotspot regimes, while (b) and (d) show less degree of transience of hotspots. 

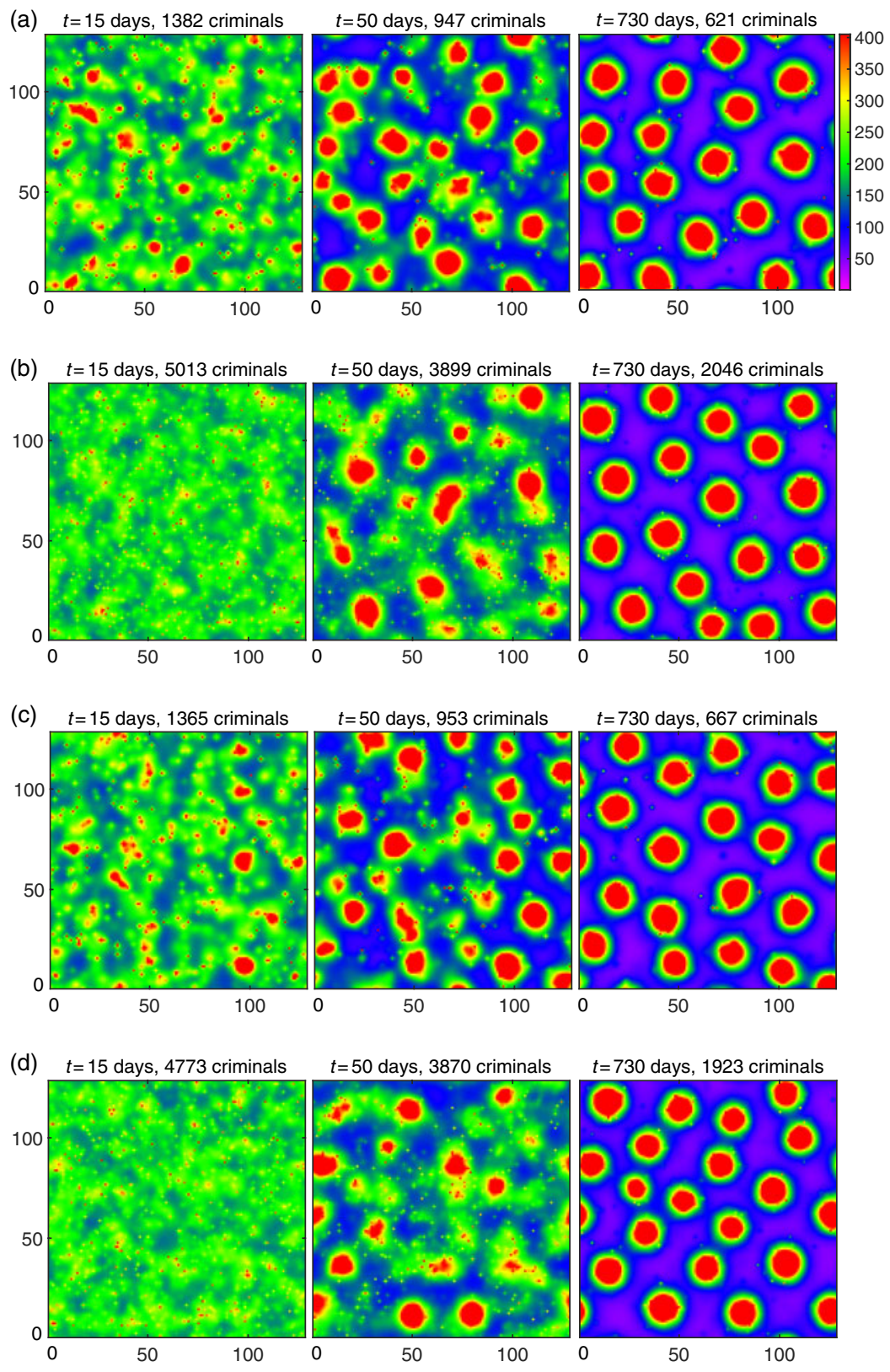

FIGURE 4. Plot of the attractiveness $A_{\mathbf{s}}^{\ell}(t)$ for the SSRB model [104] and the SSRB-IPC model. For both models, the parameters and initial conditions (at $t=0$ ) are set as $L=128, \ell=1, D=100, \omega=1 / 15, \eta=$ $0.03, A 0=1 / 30, n 0_{\mathrm{s}}^{\ell} \cong \bar{n}^{\ell}$, and $B 0^{\ell}$ is set to be $\bar{B}$ except for sites with slight perturbations. For the SSRB model [104], in (a) and (b), we set $\Gamma=0.0019 q, \theta=5.6 / q, q=10$ in (a), and $q=10 \sqrt{10}$ in (b). For the SSRB-IPC model, in (c) and (d), we set $\Gamma=0.0019 q, \theta=5.6 / q, q=10$ in (c), and $q=10 \sqrt{10}$ in (d). (a) and (c) show the stationary hotspots regimes. (b) and (d) show even less degree of transience of hotspots. 


\section{Analysis and discussion}

\subsection{Martingale formulation}

The Martingale formulation of a Markov pure jump process characterises the process as sum of an integral part involving the infinitesimal mean and a Martingale part involving the infinitesimal variance. For every $t$, we define $\left(B^{\ell}(t), n^{\ell}(t)\right):=\left\{\left(B_{\mathbf{s}}^{\ell}(t), n_{\mathbf{s}}^{\ell}(t)\right): \mathbf{s} \in \mathscr{S}^{\ell}\right\}$. In a similar way, we can define the stochastic processes $n^{\ell}(t), B^{\ell}(t)$ and $A^{\ell}(t)$ associated with the SSRB-IPC model. For arbitrary scalar fields $f^{\ell}:=\left\{f_{\mathbf{s}}^{\ell}: \mathbf{s} \in \mathscr{S}^{\ell}\right\}$ and $g^{\ell}:=\left\{g_{\mathbf{s}}^{\ell}: \mathbf{s} \in \mathscr{S}^{\ell}\right\}$, we define the discrete inner product and $L^{p}$ norm over the lattice $\mathscr{S}^{\ell}$ :

$$
\left\langle f^{\ell}, g^{\ell}\right\rangle:=\ell^{2} \sum_{\mathbf{s} \in \mathscr{S}^{\ell}} f_{\mathbf{s}}^{\ell} g_{\mathbf{s}}^{\ell}, \quad\left|f^{\ell}\right|_{p}:=\left(\ell^{2} \sum_{\mathbf{s} \in \mathscr{S}^{\ell}}\left|f_{\mathbf{s}}^{\ell}\right|^{p}\right)^{1 / p}, \quad p \geqslant 1 .
$$

Let $\phi^{\ell}=\left\{\phi_{\mathbf{s}}^{\ell}: \mathbf{s} \in \mathscr{S}^{\ell}\right\}$ be an arbitrary stationary scalar field, and we define

$$
\left\langle\left(B^{\ell}(t), n^{\ell}(t)\right), \phi^{\ell}\right\rangle:=\left(\left\langle B^{\ell}(t), \phi^{\ell}\right\rangle,\left\langle n^{\ell}(t), \phi^{\ell}\right\rangle\right) .
$$

As $\left\langle\left(B^{\ell}(t), n^{\ell}(t)\right), \phi^{\ell}\right\rangle$ is a Markov pure jump process with state space $\mathbb{R}^{2}$, a Martingale approach is applicable (e.g., $[20,21,54,65,96])$ and a Martingale formulation can be derived as follows:

Theorem 3.1 For each fixed $\ell$, before the possible blow-up time, $\left\langle\left(B^{\ell}(t), n^{\ell}(t)\right), \phi^{\ell}\right\rangle$ can be written as

$$
\left\{\begin{aligned}
\left\langle B^{\ell}(t), \phi^{\ell}\right\rangle= & \left\langle B 0^{\ell}, \phi^{\ell}\right\rangle+\int_{0}^{t} \mathscr{G}_{1}^{\ell}\left(\left\langle\left(B^{\ell}(r), n^{\ell}(r)\right), \phi^{\ell}\right\rangle\right) d r \\
& +\mathscr{M}_{1}^{\ell}\left(\left\langle\left(B^{\ell}(t), n^{\ell}(t)\right), \phi^{\ell}\right\rangle\right), \\
\left\langle n^{\ell}(t), \phi^{\ell}\right\rangle= & \left\langle n 0^{\ell}, \phi^{\ell}\right\rangle+\int_{0}^{t} \mathscr{G}_{2}^{\ell}\left(\left\langle\left(B^{\ell}(r), n^{\ell}(r)\right), \phi^{\ell}\right\rangle\right) d r \\
& +\mathscr{M}_{2}^{\ell}\left(\left\langle\left(B^{\ell}(t), n^{\ell}(t)\right), \phi^{\ell}\right\rangle\right),
\end{aligned}\right.
$$

where $\mathscr{M}_{i}^{\ell}\left(\left\langle\left(B^{\ell}(t), n^{\ell}(t)\right), \phi^{\ell}\right\rangle\right), i=1,2$, are Martingales that start at $t=0$ as zeros, and $\mathscr{G}_{i}^{\ell}\left(\left\langle\left(B^{\ell}(t), n^{\ell}(t)\right), \phi^{\ell}\right\rangle\right), i=1,2$, are the infinitesimal means for the attractiveness and the burglar distribution, respectively, and

$$
\begin{aligned}
& \mathscr{G}_{1}^{\ell}\left(\left\langle\left(B^{\ell}(t), n^{\ell}(t)\right), \phi^{\ell}\right\rangle\right) \\
& =\left\langle\frac{\eta D}{4} \Delta^{\ell} B^{\ell}(t)-\omega B^{\ell}(t)+\theta A^{\ell}(t) n^{\ell}(t), \phi^{\ell}\right\rangle, \\
& \mathscr{G}_{2}^{\ell}\left(\left\langle\left(B^{\ell}(t), n^{\ell}(t)\right), \phi^{\ell}\right\rangle\right) \\
& =\ell^{2} \sum_{\boldsymbol{s} \in \mathscr{S}}\left[-n_{s}^{\ell}(t) A_{s}^{\ell}(t)+D \ell^{-2}\left(A_{s}^{\ell} \sum_{\boldsymbol{s}^{\prime} \sim \boldsymbol{s}} \frac{n_{s^{\prime}}^{\ell}(t)}{T_{\boldsymbol{s}^{\prime}}^{\ell}(t)}-n_{\boldsymbol{s}}^{\ell}(t)\right)+\Gamma\right] \phi_{s}^{\ell} .
\end{aligned}
$$

The variances of $\mathscr{M}_{i}^{\ell}\left(\left\langle\left(B^{\ell}(t), n^{\ell}(t)\right), \phi^{\ell}\right\rangle\right), i=1,2$, can be characterised in the following way:

$$
\operatorname{Var}\left(\mathscr{M}_{i}^{\ell}\left(\left\langle\left(B^{\ell}(t), n^{\ell}(t)\right), \phi^{\ell}\right\rangle\right)\right)=\int_{0}^{t} \mathbb{E}\left[\mathscr{V}_{i}^{\ell}\left(\left\langle\left(B^{\ell}(r), n^{\ell}(r)\right), \phi^{\ell}\right\rangle\right)\right] d r, \quad i=1,2,
$$


where $\mathscr{V}_{i}^{\ell}\left(\left\langle\left(B^{\ell}(t), n^{\ell}(t)\right), \phi^{\ell}\right\rangle\right), i=1,2$, are the infinitesimal variances for the attractiveness and the burglar distribution, respectively, and

$$
\begin{aligned}
& \mathscr{V}_{1}^{\ell}\left(\left\langle\left(B^{\ell}(t), n^{\ell}(t)\right), \phi^{\ell}\right\rangle\right) \\
& =\ell^{2}\left\langle\theta^{2} n^{\ell}(t) A^{\ell}(t)+\frac{\ell^{-\sigma} \omega^{2}}{D} B^{\ell}(t)^{2}+\frac{D \ell^{2} \eta^{2}}{16}\left(\Delta^{\ell} B^{\ell}(t)\right)^{2},\left(\phi^{\ell}\right)^{2}\right\rangle, \\
& \mathscr{V}_{2}^{\ell}\left(\left\langle\left(B^{\ell}(t), n^{\ell}(t)\right), \phi^{\ell}\right\rangle\right) \\
& =\ell^{2}\left\langle A^{\ell}(t) n^{\ell}(t)+\Gamma,\left(\phi^{\ell}\right)^{2}\right\rangle+D \ell^{4} \sum_{\boldsymbol{s} \in \mathscr{S}} n_{s}^{\ell}(t)\left(\sum_{s^{\prime} \sim s} \frac{A_{s^{\prime}}^{\ell}(t)}{T_{s}^{\ell}\left(t^{-}\right)}\left|\nabla_{s \rightarrow s^{\prime}}^{\ell} \phi^{\ell}\right|^{2}\right),
\end{aligned}
$$

where $\nabla_{s \rightarrow s^{\prime}}^{\ell} \phi_{s}^{\ell}$ denotes the discrete directional derivative from $s$ pointing towards $s^{\prime}$, that is, $\nabla_{s \rightarrow s^{\prime}}^{\ell} \phi_{s}^{\ell}=\left(\phi_{s}^{\ell}-\phi_{s^{\prime}}^{\ell}\right) / \ell$.

Proof of Theorem 3.1 We compute the infinitesimal means and variances for the Markov pure jump process $\left\langle\left(B^{\ell}(t), n^{\ell}(t)\right), \phi^{\ell}\right\rangle$ for a fixed $\ell$, using the methods in $[1,17,22,44,46,53,62,73$, $74,83,85]$. In the computational steps, we will drop the superscript $\ell$ for simplicity.

As $\mathscr{G}_{1}^{\ell}\left(\left\langle\left(B^{\ell}(t), n^{\ell}(t)\right), \phi^{\ell}\right\rangle\right)$ is the infinitesimal mean for $\left\langle B^{\ell}\left(t^{-}\right), \phi^{\ell}\right\rangle$, from (2.6), we have

$$
\begin{aligned}
& \mathscr{G}_{1}\left(\left\langle B^{\ell}\left(t^{-}\right), \phi^{\ell}\right\rangle,\left\langle n^{\ell}\left(t^{-}\right), \phi^{\ell}\right\rangle\right) \\
& =\ell^{2} \theta \sum_{\mathbf{s} \in \mathscr{S}} n_{\mathbf{s}}\left(t^{-}\right) A_{\mathbf{s}}\left(t^{-}\right) \phi_{\mathbf{s}}-D \ell^{\sigma+2} \sum_{\mathbf{s} \in \mathscr{S}} \frac{\omega}{D \ell^{\sigma}} \phi_{\mathbf{s}} B_{\mathbf{s}}\left(t^{-}\right)+D \ell^{2} \sum_{\mathbf{s} \in \mathscr{S}} \frac{\eta}{4} \Delta_{\mathbf{s}} B_{\mathbf{s}}\left(t^{-}\right) \phi_{\mathbf{s}} \\
& =\ell^{2} \sum_{\mathbf{s} \in \mathscr{S}}\left[\frac{\eta D}{4} \Delta B_{\mathbf{s}}\left(t^{-}\right)-\omega B_{\mathbf{s}}\left(t^{-}\right)+\theta A_{\mathbf{s}}\left(t^{-}\right) n_{\mathbf{s}}\left(t^{-}\right)\right] \phi_{\mathbf{s}} .
\end{aligned}
$$

From (3.8), we obtain (3.3).

As $\mathscr{G}_{2}^{\ell}\left(\left\langle\left(B^{\ell}(t), n^{\ell}(t)\right), \phi^{\ell}\right\rangle\right)$ is the infinitesimal mean for $\left\langle n^{\ell}\left(t^{-}\right), \phi^{\ell}\right\rangle$, from (2.2), we have

$$
\begin{aligned}
& \mathscr{G}_{2}\left(\left\langle\left(B^{\ell}\left(t^{-}\right), n^{\ell}\left(t^{-}\right)\right), \phi^{\ell}\right\rangle\right) \\
& =-\ell^{2} \sum_{\mathbf{s} \in \mathscr{S}} n_{\mathbf{s}}\left(t^{-}\right) \phi_{\mathbf{s}} A_{\mathbf{s}}\left(t^{-}\right)+D \sum_{\mathbf{s} \in \mathscr{S}} n_{\mathbf{s}}\left(t^{-}\right)\left(\sum_{\mathbf{s}^{\prime} \sim \mathbf{s}} \frac{A_{\mathbf{s}^{\prime}}\left(t^{-}\right)}{T_{\mathbf{s}}\left(t^{-}\right)}\left(\phi_{\mathbf{s}^{\prime}}-\phi_{\mathbf{s}}\right)\right)+\Gamma \ell^{2} \sum_{\mathbf{s} \in \mathscr{S}} \phi_{\mathbf{s}} \\
& =-\ell^{2} \sum_{\mathbf{s} \in \mathscr{S}} n_{\mathbf{s}}\left(t^{-}\right) \phi_{\mathbf{s}} A_{\mathbf{s}}\left(t^{-}\right)+D \sum_{\mathbf{s} \in \mathscr{S}} \phi_{\mathbf{s}}\left(A_{\mathbf{s}}\left(t^{-}\right) \sum_{\mathbf{s}^{\prime} \sim \mathbf{s}} \frac{n_{\mathbf{s}^{\prime}}\left(t^{-}\right)}{T_{\mathbf{s}^{\prime}}\left(t^{-}\right)}-n_{\mathbf{s}}\left(t^{-}\right)\right)+\Gamma \ell^{2} \sum_{\mathbf{s} \in \mathscr{S}} \phi_{\mathbf{s}}
\end{aligned}
$$

From (3.9), we obtain (3.4).

As $\mathscr{V}_{1}^{\ell}\left(\left\langle\left(B^{\ell}(t), n^{\ell}(t)\right), \phi^{\ell}\right\rangle\right)$ is the infinitesimal variance of $\left\langle B^{\ell}\left(t^{-}\right), \phi^{\ell}\right\rangle$, we have

$$
\begin{aligned}
& \mathscr{V}_{1}^{\ell}\left(\left\langle\left(B^{\ell}(t), n^{\ell}(t)\right), \phi^{\ell}\right\rangle\right) \\
& =\lim _{\delta t \rightarrow 0} \frac{1}{\delta t} \mathbb{E}\left[\left(\left\langle B\left(\delta t+t^{-}\right), \phi\right\rangle-\left\langle B\left(t^{-}\right), \phi\right\rangle\right)^{2} \mid\left(B\left(t^{-}\right), n\left(t^{-}\right)\right)\right]
\end{aligned}
$$




$$
\begin{aligned}
& =\ell^{4} \theta^{2} \sum_{\mathbf{s} \in \mathscr{S}} n_{\mathbf{s}}\left(t^{-}\right) A_{\mathbf{s}}\left(t^{-}\right) \phi_{\mathbf{s}}^{2}+\ell^{4} D \ell^{\sigma} \sum_{\mathbf{s} \in \mathscr{S}} \ell^{-2 \sigma} \frac{\omega^{2}}{D^{2}} B_{\mathbf{s}}\left(t^{-}\right)^{2} \phi_{\mathbf{s}}^{2}+D \ell^{6} \sum_{\mathbf{s} \in \mathscr{S}} \frac{\eta^{2}}{16}\left(\Delta_{\mathbf{s}} B_{\mathbf{s}}\left(t^{-}\right)\right)^{2} \phi_{\mathbf{s}}^{2} \\
& =\ell^{2} \sum_{\mathbf{s} \in \mathscr{S}}\left[\ell^{2} \theta^{2} n_{\mathbf{s}}\left(t^{-}\right) A_{\mathbf{s}}\left(t^{-}\right)+\frac{\ell^{2-\sigma} \omega^{2}}{D} B_{\mathbf{s}}\left(t^{-}\right)^{2}+\frac{D \ell^{4} \eta^{2}}{16}\left(\Delta_{\mathbf{s}} B_{\mathbf{s}}\left(t^{-}\right)\right)^{2}\right] \phi_{\mathbf{s}}^{2} .
\end{aligned}
$$

From (3.10), we obtain (3.6).

As $\mathscr{V}_{2}^{\ell}\left(\left\langle\left(B^{\ell}(t), n^{\ell}(t)\right), \phi^{\ell}\right\rangle\right)$ is the infinitesimal variance of $\left\langle n^{\ell}\left(t^{-}\right), \phi^{\ell}\right\rangle$, we have

$$
\begin{aligned}
& \mathscr{V}_{2}^{\ell}\left(\left\langle\left(B^{\ell}(t), n^{\ell}(t)\right), \phi^{\ell}\right\rangle\right) \\
& =\lim _{\delta t \rightarrow 0} \frac{1}{\delta t} \mathbb{E}\left[\left(\left\langle n\left(\delta t+t^{-}\right), \phi\right\rangle-\left\langle n\left(t^{-}\right), \phi\right\rangle\right)^{2} \mid\left(B\left(t^{-}\right), n\left(t^{-}\right)\right)\right] \\
& =\ell^{4} \sum_{\mathbf{s} \in \mathscr{S}} A_{\mathbf{s}}\left(t^{-}\right) n_{\mathbf{s}}\left(t^{-}\right) \phi_{\mathbf{s}}^{2}+D \ell^{2} \sum_{\mathbf{s} \in \mathscr{S}} n_{\mathbf{s}}\left(t^{-}\right)\left(\sum_{\mathbf{s}^{\prime} \sim \mathbf{s}} \frac{A_{\mathbf{s}^{\prime}}\left(t^{-}\right)}{T_{\mathbf{s}}\left(t^{-}\right)}\left(\phi_{\mathbf{s}^{\prime}}-\phi_{\mathbf{s}}\right)^{2}\right)+\Gamma \ell^{4} \sum_{\mathbf{s} \in \mathscr{S}} \phi_{\mathbf{s}}^{2} \\
& =\ell^{4} \sum_{\mathbf{s} \in \mathscr{S}}\left[A_{\mathbf{s}}\left(t^{-}\right) n_{\mathbf{s}}\left(t^{-}\right) \phi_{\mathbf{s}}^{2}+D n_{\mathbf{s}}\left(t^{-}\right)\left(\sum_{\mathbf{s}^{\prime} \sim \mathbf{s}} \frac{A_{\mathbf{s}^{\prime}}\left(t^{-}\right)}{T_{\mathbf{s}}\left(t^{-}\right)}\left|\nabla_{\mathbf{s} \rightarrow \mathbf{s}^{\prime}} \phi\right|^{2}\right)+\Gamma \phi_{\mathbf{s}}^{2}\right] .
\end{aligned}
$$

From (3.11), we obtain (3.7).

With the infinitesimal means and variances obtained, we apply Theorem (1.6), [20] or Theorem 3.32, [65], to obtain (3.2) and apply Exercise 3.8.12 of [8] to obtain (3.5). We can also apply Lemma A 1.5.1 in [54] or Proposition B.1 in [79] to obtain (3.5). The proof of Theorem 3.1 is completed.

\subsection{Continuum analogue of the SSRB-IPC model}

With a similar derivation of the hydrodynamic limit of interacting particle systems $[26,40,54$, $55,96,97,102,103]$, we can find a continuum analogue of the agent-based SSRB-IPC model based on the Martingale formulation (3.2), where the infinitesimal variances have an equal or lower order of magnitude than $\ell$.

By (3.6) and (3.7), as $\sigma<0$, we obtain

$$
\begin{aligned}
& \mathscr{V}_{1}^{\ell}\left(\left\langle\left(B^{\ell}(t), n^{\ell}(t)\right), \phi^{\ell}\right\rangle\right) \cong \ell^{2}\left\langle\theta^{2} n^{\ell}(t) A^{\ell}(t),\left(\phi^{\ell}\right)^{2}\right\rangle+o\left(\ell^{2}\right) \cong O\left(\ell^{2}\right), \\
& \mathscr{V}_{2}^{\ell}\left(\left\langle\left(B^{\ell}(t), n^{\ell}(t)\right), \phi^{\ell}\right\rangle\right) \\
& \cong \ell^{2}\left\langle\sum_{\mathbf{s} \in \mathscr{S}} A^{\ell}(t) n^{\ell}(t)+\Gamma,\left(\phi^{\ell}\right)^{2}\right\rangle+D \ell^{2}\left\langle n_{\mathbf{s}}^{\ell}(t), \sum_{\mathbf{s}^{\prime} \sim \mathbf{s}} \frac{A_{\mathbf{s}^{\prime}}^{\ell}(t)}{T_{\mathbf{s}}^{\ell}\left(t^{-}\right)}\left|\nabla_{\mathbf{s} \rightarrow \mathbf{s}^{\prime}}^{\ell} \phi^{\ell}\right|^{2}\right\rangle \\
& \cong O\left(\ell^{2}\right) .
\end{aligned}
$$

Therefore, for $\ell$ small, it is reasonable to set the continuum version of the infinitesimal mean vector as a continuum analogue of the SSRB-IPC model, and by (3.3) and (3.4) we obtain

$$
\left\{\begin{aligned}
\frac{\partial B}{\partial t} & =\frac{\eta D}{4} \Delta B-\omega B+\theta n A, \\
\frac{\partial n}{\partial t} & =\frac{D}{4} \vec{\nabla} \cdot\left(\vec{\nabla} n-\frac{2 n}{A} \vec{\nabla} A\right)-n A+\Gamma, \\
n(0) & =n 0, B(0)=B 0,
\end{aligned}\right.
$$




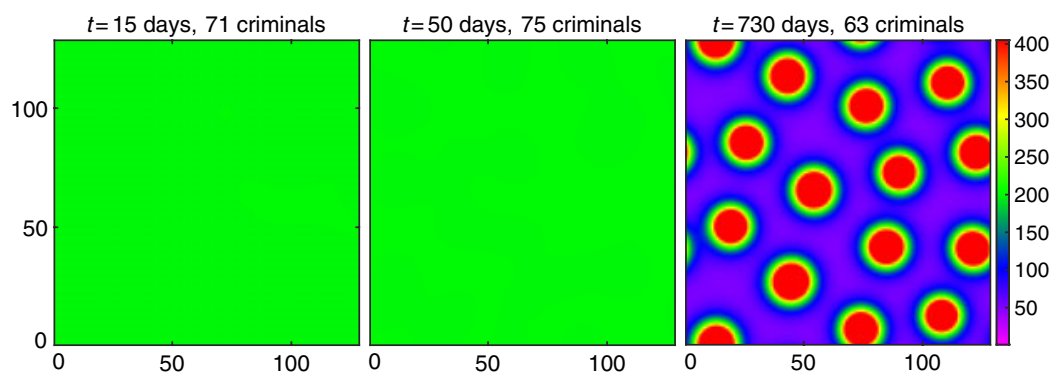

FIGURE 5. Output of the attractiveness $A(\mathbf{x}, t)$ for the continuum analogue of the SSRB model with hotspot formation. The parameters and data are the equivalent of those used to create plots in Figures 3 (a)-(d) and 4 (a)-(d).

where $n(\mathbf{x}, t), A(\mathbf{x}, t)$ and $B(\mathbf{x}, t), \mathbf{x} \in \mathscr{D}$, are the continuum versions of $n_{\mathbf{s}}^{\ell}(t), A_{\mathbf{s}}^{\ell}(t)$ and $B_{\mathbf{s}}^{\ell}(t)$, respectively. The continuum analogue of the SSRB-IPC model (3.14), the DTS model [93] ((3.2) and (3.5) in [93]) and the SSRB model ((18) in [104]) is the same.

We check the validity of the continuum equation of the agent-based SSRB-IPC model through simulations. We use the same algorithm as that for the continuum analogue of the DTS model [93] (see (3.11)-(3.13) in [93]). Figure 5 shows example output of the attractiveness $A(\mathbf{x}, t)$ in the cases of hotspot formation. The same colour key is used as in Figure 1. We infer from (3.14) that the parameters and data used to create Figures 3(a), (b), (c) and (d), and 4(a), (b), (c) and (d) give rise to the same attractiveness field for the continuum analogue of the SSRB-IPC model. Hence, we only display the output once here in Figure 5. For the cases of zero hotspot formation, the parameters and data used to create Figures 1(a), (b), (c) and (d) and 2(a), (b), (c) and (d) give rise to the same attractiveness field for the continuum analogue of the SSRB-IPC model, which is the equilibrium as the continuum system stays at the equilibrium with equilibrium initial data.

The regimes of dynamic hotspots are absent in the continuum simulations. As the total burglar population decreases, deviations of behavioural regimes of the SSRB-IPC model from its continuum equation increase. These observations are closely linked to the finite size effects exhibited in Figures 1, 2, 3, and 4. The same hotspot pattern formations are observed in the simulations of the DTS model [93], the SSRB model [104] and their respective continuum equations.

\subsection{Quantification of the pattern formation}

To analyse the finite size effects mathematically, we quantify the pattern formation of hotspots by measuring the degree of hotpot transience with statistics through quantitative simulations.

\subsubsection{Statistics measuring degree of hotspot transience}

We use the same statistics as in [104] to measure degree of hotspot transience:

(i) Relative Fisher information relative to the uniform measure over $\mathscr{S}^{\ell}$, logarithm mean type (see Appendix of [16], and also [27, 28, 29]):

$$
\mathscr{I}^{\ell}(t):=\ell^{-2} \sum_{\mathbf{s} \in \mathscr{S}}\left(A_{\mathbf{s}}^{\ell}(t)-A_{\mathbf{s}^{\prime}}^{\ell}(t)\right)\left(\log A_{\mathbf{s}}^{\ell}(t)-\log A_{\mathbf{s}}^{\ell}(t)\right)
$$


(ii) Rate of change of $A^{\ell}(t)$ over time in the discrete $L^{p}$ norm, $p \geqslant 1$. Fixing a time increment $\Delta t>0$, we define the $\mathrm{L}^{p}$-area rate of change as

$$
\delta_{p}^{\ell}(t):=\left|A^{\ell}(t+\Delta t)-A^{\ell}(t)\right|_{p} .
$$

(iii) Rate of change of the area of regions with attractiveness higher than $2 \bar{A}$ (red regions). For these regions, we define the relative overlapping area $\mathscr{O}^{l}(t)$ and non-overlapping area $\mathscr{N}^{\ell}(t)$ as follows:

$$
\begin{aligned}
& \mathscr{O}^{\ell}(t):=\frac{1}{A_{R}^{\ell}} \sum_{\substack{\mathbf{s} \in \mathscr{S}^{\ell} \\
A_{\mathbf{s}}^{\ell}(t) \geqslant 2 \bar{A} \\
A_{\mathbf{s}}^{\ell}(t+\Delta t) \geqslant 2 \bar{A}}} \mathbf{1}(\mathbf{s}), \\
& \mathscr{N}^{\ell}(t):=\frac{1}{A_{R}^{\ell}}\left[\sum_{\substack{\mathbf{s} \in \mathscr{S}^{\ell} \\
A_{\mathbf{s}}^{\ell}(t+\Delta t) \geqslant 2 \bar{A}}} \mathbf{1}(\mathbf{s})+\sum_{\substack{\mathbf{s} \in \mathscr{S}^{\ell} \\
A_{\mathbf{s}}^{\ell}(t) \geqslant 2 \bar{A}}} \mathbf{1}(\mathbf{s})\right]-2 \mathscr{O}^{\ell}(t),
\end{aligned}
$$

where $\mathbf{1}(\mathbf{s})$ is an indicator function, and $A_{R}^{\ell}$ is the renormalisation:

$$
A_{R}^{\ell}=\sum_{\substack{\mathbf{s} \in \mathscr{S}^{\ell} \\ A_{\mathbf{s}}^{\ell}(t) \geqslant 2 \bar{A}}} \mathbf{1}(\mathbf{s}) .
$$

For the continuum equation (3.13), in a very similar way, we can define all the analogues of the above quantities, which will be denoted as $\mathscr{I}(t), \delta_{p}(t), \mathscr{O}(t)$ and $\mathscr{N}(t)$.

\subsubsection{Numerical simulations of the statistics}

Example output of direct simulations for the statistics (3.15)-(3.18) for the agent-based SSRB model [104] and the agent-based SSRB-IPC model can be found in Figures 6, 7, 8 and 9. All the simulations are run with $\Delta t=10, t \in[0,730]$ and $p=1$. The blue, magenta, black and red lines show results with increasing values of $q$ and thus increasing burglar population.

For the agent-based SSRB model [104], Figures 6(a) and 7(a) show results with zero hotspot formation, and the blue, magenta, black and red lines represent the results associated with the simulations in Figures 1(a), (b), 2(a) and (b), respectively. Figures 6(b), 7(b), 8(a) and 9(a) show the results associated with hotspot formation regimes, and the blue, magenta, black red and green lines represent results associated with the simulations in Figures 3(a), (b), 4(a), (b) and 5 , respectively.

For the agent-based SSRB-IPC model, Figures 6(c) and 7(c) show results with zero hotspot formation, and the blue, magenta, black and red lines represent the results associated with the simulations in Figures 1(c), (d), 2(c) and (d). Figures 6(d), 7(d), 8(b) and 9(b) show the results associated with hotspot formation regimes, and the blue, magenta, black, red and green lines represent the results associated with the simulations in Figures 3(c), (d), 4(c), (d) and 5, respectively.

As in [104], the simulation output shows that a larger degree of hotspot transience appears with a smaller burglar population. Also, the continuum simulations exhibit the lowest degree of 


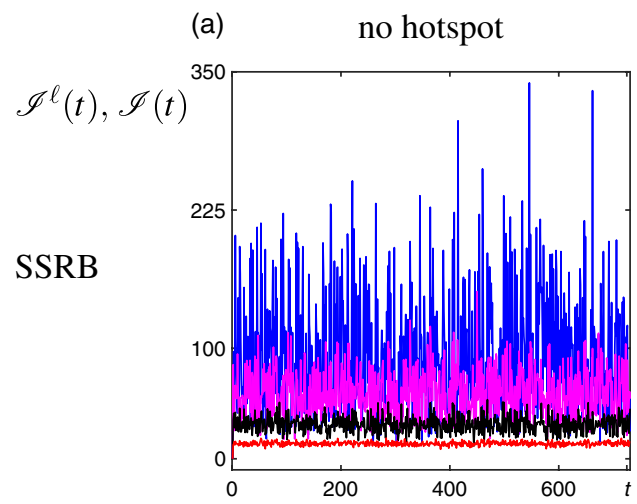

(b) hotspot

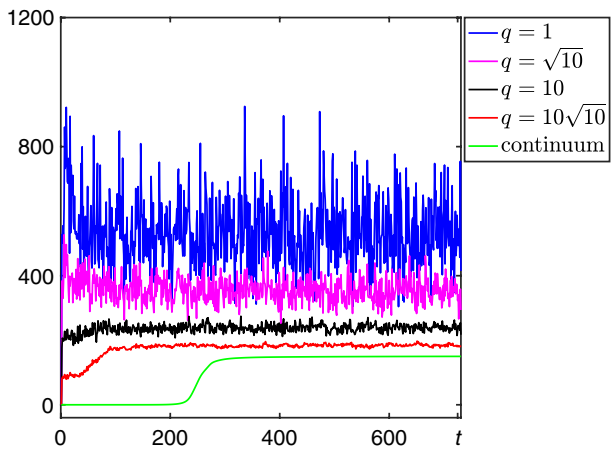

(c)

(d)
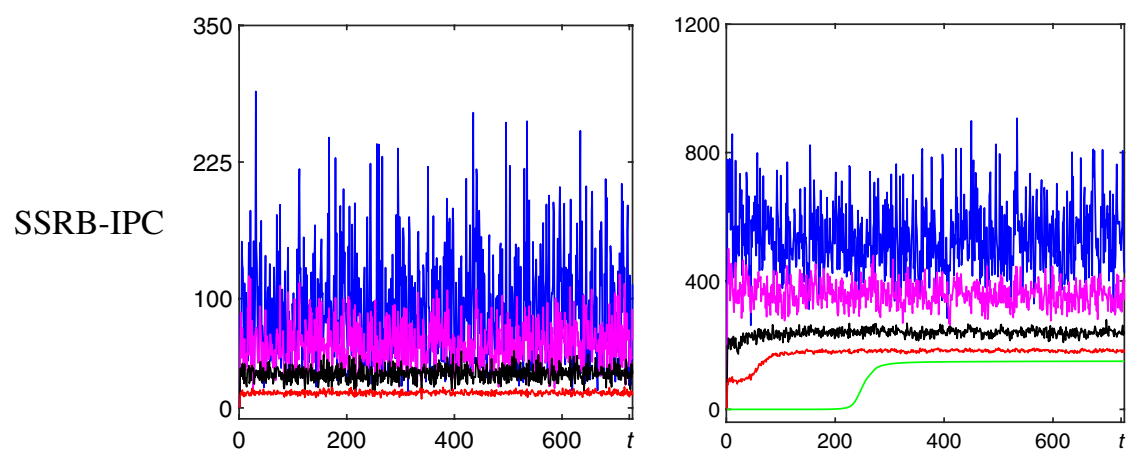

FIGURE 6. Examples of the relative Fisher information $\mathscr{I}^{\ell}(t)$ and $\mathscr{I}(t)$ for zero hotspot formation and hotspot formation. For the SSRB model [104], (a) shows results with cases of zero hotspot formation, and the blue, magenta, black and red lines represent the statistics of the models plotted in Figures 1(a), (b), 2(a) and (b), respectively. (b) shows results associated with the hotspot formation regimes, and the blue, magenta, black, red and green lines show results with the simulations in Figures 3(a), (b), 4(a), (b) and 5, respectively. For the SSRB-IPC model, (c) shows results with cases of zero hotspot formation, and the blue, magenta, black and red lines represent the statistics of the models plotted in Figures 1(c), (d), 2(c) and (d), respectively. (d) shows results associated with the hotspot formation regimes, and the blue, magenta, black, red and green lines show results with the simulations in Figures 3(c), (d), 4(c), (d) and 5, respectively.

hotspot transience. The peaks in Figures 7(b), (d), 9(a) and (b) correspond to the initial emergence of hotspots in the SSRB model, the SSRB-IPC model, and their continuum equations. The statistics increase during the emergence period as hotspots form, and decrease and stabilise (or directly stabilise) as hotspots stabilise. The same simulation output is also observed over other random paths. The output matches well with the qualitative simulations in Figures 1, 2, 3, 4, and 5, which indicates that the above statistics are suitable to use.

\subsection{Mathematical analysis and simulations of the finite size effects}

With quantification of the degree of hotspot transience, we analyse the finite size effects based on the Martingale formulation, and simulations are run which supports our theoretical conclusion. 

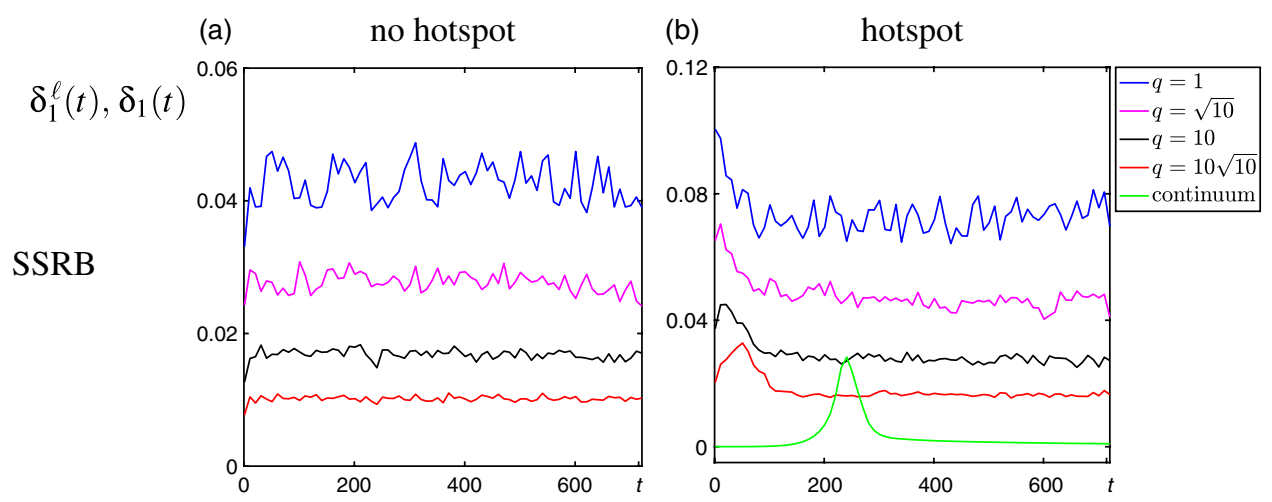

(c)

(d)
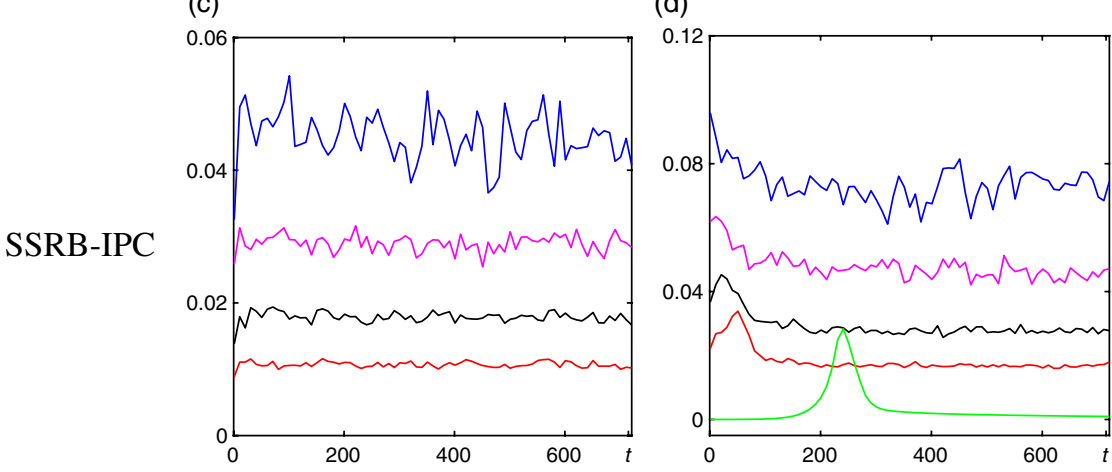

FIGURE 7. Examples of the $\mathrm{L}^{1}$-area rate of change $\delta_{1}^{\ell}(t)$ and $\delta_{1}(t)$ for SSRB and SSRB-IPC models for zero hotspot formation and hotspot formation. For the SSRB model [104], (a) shows results with cases of zero hotspot formation, and the blue, magenta, black and red lines represent the statistics of the models plotted in Figures 1(a), (b), 2(a) and (b), respectively. (b) shows results associated with the hotspot formation regimes, and the blue, magenta, black, red and green lines show results with the simulations in Figures 3(a), (b), 4(a), (b) and 5, respectively. For the SSRB-IPC model, (c) shows results with cases of zero hotspot formation, and the blue, magenta, black and red lines represent the statistics of the models plotted in Figures 1(c), (d), 2(c) and (d), respectively. (d) shows results associated with the hotspot formation regimes, and the blue, magenta, black, red and green lines show results with the simulations in Figures 3(c), (d), 4(c), (d) and 5, respectively.

\subsubsection{A theory of the finite size effects}

We analyse the deterministic and stochastic component of the Martingale formulation with varying initial burglar number, repeat victimisation strength $\theta$ and replacement rate $\Gamma$. Fixing $\Theta>0$, $\mathfrak{d}>0$, for $q \in\left(0, D / \ell^{2} \mathfrak{d}\right)$, we consider the agent-based SSRB-IPC model with parameters and initial data scaled in the following way:

$$
\left(B^{\ell,(q)}(t), n^{\ell,(q)}(t)\right):=\left.\left(B^{\ell}(t), n^{\ell}(t)\right)\right|_{B 0^{\ell} \cong \overline{\mathfrak{B}}, n 0^{\ell} \cong q \overline{\mathfrak{n}}^{\ell}, \theta=\frac{\Theta}{q}, \Gamma=q \mathfrak{d}} \cdot
$$

Here, $\overline{\mathfrak{B}}, \overline{\mathfrak{n}}^{\ell}$ are the homogeneous equilibrium values as in (2.7) with $\theta=\Theta$ and $\Gamma=\mathfrak{d}$ :

$$
\overline{\mathfrak{B}}=\frac{\Theta \mathfrak{d}}{\omega}, \quad \overline{\mathfrak{n}}^{\ell}=\frac{\mathfrak{d} \ell^{2}}{D\left(1-e^{-\ell^{2}(\overline{\mathfrak{B}}+A 0) D^{-1}}\right)} \cong \frac{\mathfrak{d}}{\overline{\mathfrak{B}}+A 0} .
$$


(a)

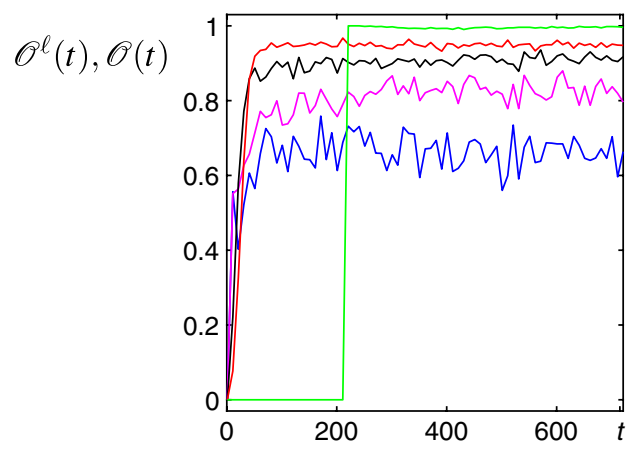

(b) SSRB-IPC

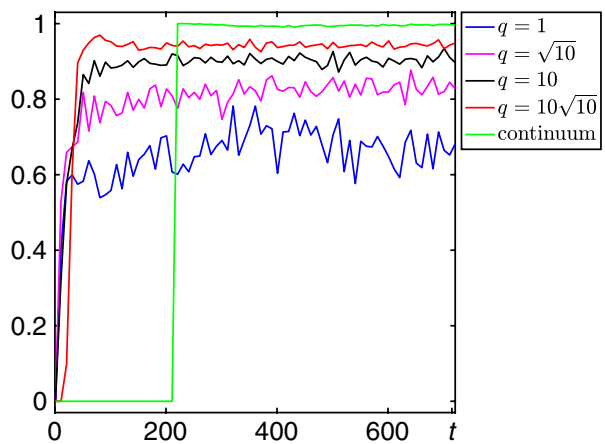

FIGURE 8. Examples of the relative overlapping area, $\mathscr{O}^{\ell}(t)$ and $\mathscr{O}(t)$, associated with hotspot formation regimes. For the SSRB model [104], in (a), the blue, magenta, black, red and green lines represent the statistics of the models plotted in Figures 3(a), (b), 4(a), (b) and 5, respectively. For the SSRB-IPC model, in (b), the blue, magenta, black, red and green lines show results with the simulations in Figures 3(a), (b), $4(\mathrm{a})$, (b) and 5, respectively.
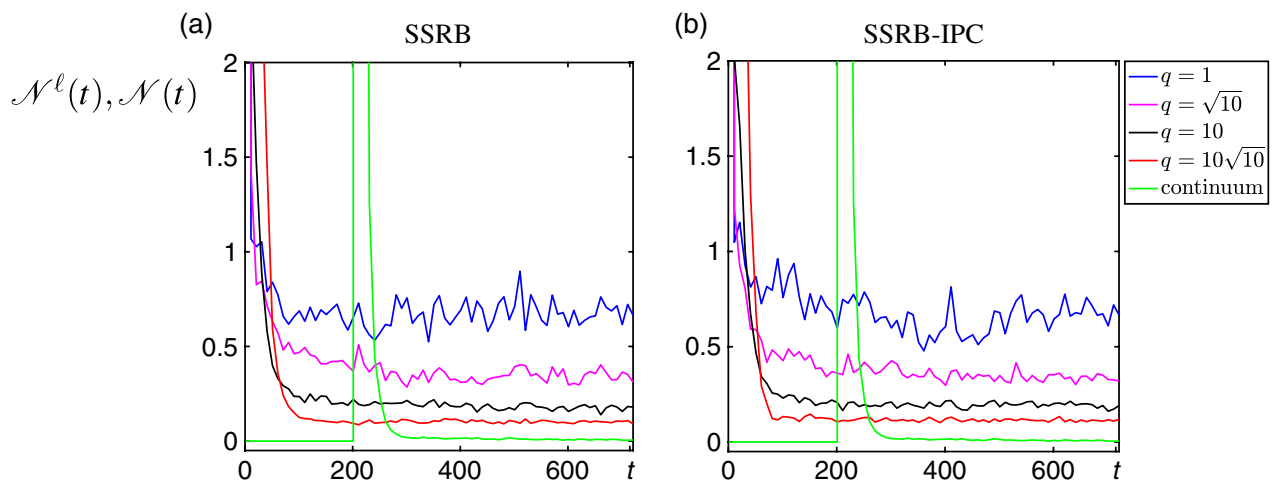

FIGURE 9. Examples of the relative non-overlapping area, $\mathscr{N}^{\ell}(t)$ and $\mathscr{N}(t)$, associated with hotspot formation regimes. For the SSRB model [104], in (a), the blue, magenta, black, red and green lines represent the statistics of the models plotted in Figures 3(a), (b), 4(a), (b) and 5, respectively. For the SSRB-IPC model, in (b), the blue, magenta, black, red and green lines show results with the simulations in Figures $3(\mathrm{a}),(\mathrm{b}), 4(\mathrm{a}),(\mathrm{b})$ and 5 , respectively.

As $q$ increases, the initial burglar population and the burglar replacement rate both increase while the initial attractiveness field remains fixed. We will use the notations $\mathscr{G}_{i}^{\ell,(q)}\left(t, \phi^{\ell}\right), \mathscr{V}_{i}^{\ell,(q)}\left(t, \phi^{\ell}\right)$ and $\mathscr{M}_{i}^{\ell,(q)}\left(t, \phi^{\ell}\right)$ as short for $\mathscr{G}_{i}^{\ell}\left(\left\langle\left(B^{\ell,(q)}(t), n^{\ell,(q)}(t)\right), \phi^{\ell}\right\rangle\right), \quad \mathscr{V}_{i}^{\ell}\left(\left\langle\left(B^{\ell,(q)}(t), n^{\ell,(q)}(t)\right), \phi^{\ell}\right\rangle\right)$ and $\mathscr{M}_{i}^{\ell}\left(\left\langle\left(B^{\ell,(q)}(t), n^{\ell,(q)}(t)\right), \phi^{\ell}\right\rangle\right)$, respectively, $i=1$, 2. And applying (3.2) and (3.5) to $\left(B^{\ell,(q)}(t), n^{\ell,(q)}(t)\right)$ over a small time step $\delta t$, we obtain

$$
\left\{\begin{aligned}
\left\langle B^{\ell,(q)}(t+\delta t), \phi^{\ell}\right\rangle & =\left\langle B^{\ell,(q)}(t), \phi^{\ell}\right\rangle+\mathscr{G}_{1}^{\ell,(q)}\left(t, \phi^{\ell}\right) \delta t \\
& +\mathscr{M}_{1}^{\ell,(q)}\left(t+\Delta t, \phi^{\ell}\right)-\mathscr{M}_{1}^{\ell,(q)}\left(t, \phi^{\ell}\right), \\
\left\langle n^{\ell,(q)}(t+\delta t), \phi^{\ell}\right\rangle= & \left\langle n^{\ell,(q)}(t), \phi^{\ell}\right\rangle+\mathscr{G}_{2}^{\ell,(q)}\left(t, \phi^{\ell}\right) \delta t \\
& +\mathscr{M}_{2}^{\ell,(q)}\left(t+\Delta t, \phi^{\ell}\right)-\mathscr{M}_{2}^{\ell,(q)}\left(t, \phi^{\ell}\right) .
\end{aligned}\right.
$$


By (3.5) and additivity of the variance in time for Martingales, we have

$$
\sqrt{\operatorname{Var}\left(\mathscr{M}_{i}^{\ell,(q)}\left(t, \phi^{\ell}\right)\right)} \cong \sqrt{\mathbb{E}\left[\mathscr{V}_{i}^{\ell,(q)}\left(t, \phi^{\ell}\right)\right] \delta t}, \quad i=1,2 .
$$

This together with (3.22) implies that the infinitesimal variances are the key to estimate the standard deviation of the stochastic component, and the deviation of the trajectories of the evolution of the model from its deterministic component.

To analyse the infinitesimal variance for attractiveness, by (3.12), we have

$$
\mathscr{V}_{1}^{\ell,(q)}\left(t, \phi^{\ell}\right) \cong q^{-2} \ell^{2} \Theta^{2}\left\langle n^{\ell,(q)}(t)\left(B^{\ell,(q)}(t)+A 0\right),\left(\phi^{\ell}\right)^{2}\right\rangle .
$$

We perform estimates at the first time step. At time zero, from (3.24) and (3.21), we infer

$$
\mathscr{V}_{1}^{\ell,(q)}\left(0, \phi^{\ell}\right)=q^{-1} \ell^{2} \Theta^{2}\left\langle\overline{\mathfrak{n}}^{\ell}(\overline{\mathfrak{B}}+A 0),\left(\phi^{\ell}\right)^{2}\right\rangle=q^{-1} \ell^{2} \Theta^{2} \mathfrak{d}\left|\phi^{\ell}\right|_{2}^{2},
$$

which implies that the infinitesimal variance for the attractiveness is inversely proportional to $q$ :

$$
\mathscr{V}_{1}^{\ell,(q)}\left(0, \phi^{\ell}\right) \propto q^{-1}
$$

We have for $0<q<\tilde{q}<D$ o $/ \ell^{2}$,

$$
\mathscr{V}_{1}^{\ell,(q)}\left(0, \phi^{\ell}\right)>\mathscr{V}_{1}^{\ell,(\tilde{q})}\left(0, \phi^{\ell}\right) .
$$

This together with (3.23) implies that at the first time step we have

$$
\operatorname{Var}\left(\mathscr{M}_{1}^{\ell,(q)}\left(\delta t, \phi^{\ell}\right)\right)>\operatorname{Var}\left(\mathscr{M}_{1}^{\ell,(\tilde{q})}\left(\delta t, \phi^{\ell}\right)\right) .
$$

From (3.28) and (3.22), we infer that at the first time step a smaller value of $q$ leads to a larger deviation of the trajectory of $B^{\ell,(q)}(t)$ from its deterministic component, and the hotspots develop temporal transience in the simulations. This explains the finite size effects at the first time step. We conjecture that (3.27) remains to be true at an arbitrary later time, namely,

$$
\mathscr{V}_{1}^{\ell,(q)}\left(t, \phi^{\ell}\right)>\mathscr{V}_{1}^{\ell,(\tilde{q})}\left(t, \phi^{\ell}\right), \text { for } 0<q<\tilde{q}<\frac{D \mathfrak{d}}{\ell^{2}} \text { and } t>0,
$$

which leads to a theory of the finite size effects at an arbitrary later time.

\subsubsection{Numerical simulations of the theory of finite size effects}

To check the validity of (3.29), we perform direct simulations of $\sqrt{\mathscr{V}_{1}^{\ell,(q)}(t)}$, the infinitesimal standard deviation for the attractiveness. We make comparisons of the agent-based SSRB-IPC model with the SSRB model [104]. Example output can be found in Figure. 10. For all the simulations, we choose the test function as

$$
\phi^{\ell}(\mathbf{x})=1+\sin \left(\mathbf{x}_{1}\right) \sin \left(\mathbf{x}_{2}\right) / 20, \quad \mathbf{x}=\left(\mathbf{x}_{1}, \mathbf{x}_{2}\right) .
$$

This choice of test function is arbitrary. It is a perturbation of the constant function so that the test function is non-trival. The perturbation does not need to be periodic.

For the agent-based SSRB model [104], Figure 10(a) shows results in the cases with no hotspot formation. The blue, magenta, black and red lines show results with the simulations in Figures 1(a), (b), 2(a) and (b), respectively. Figure 10(b) shows results with hotspot formation. The 


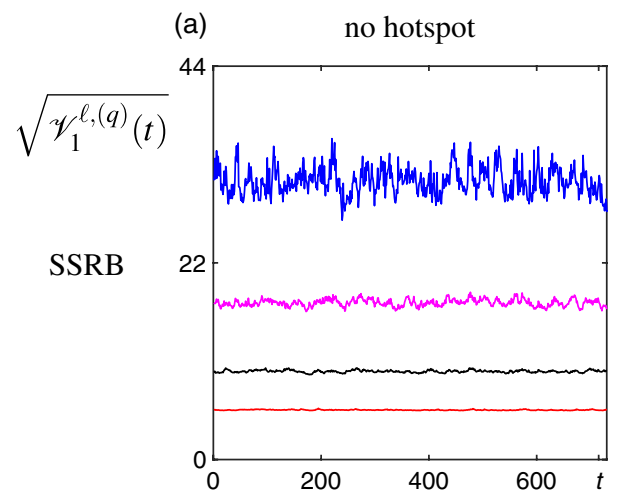

(b) hotspot

(c)

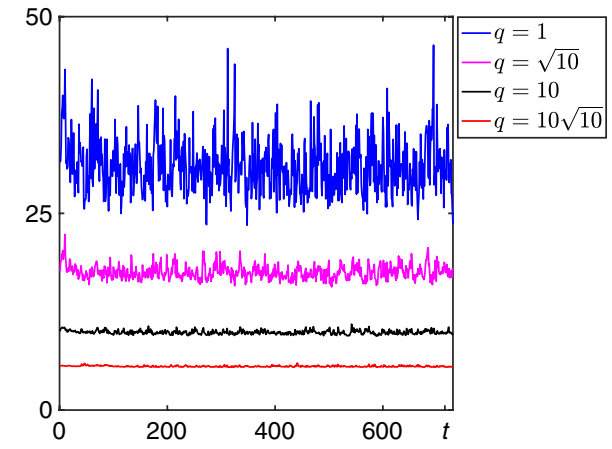

(d)
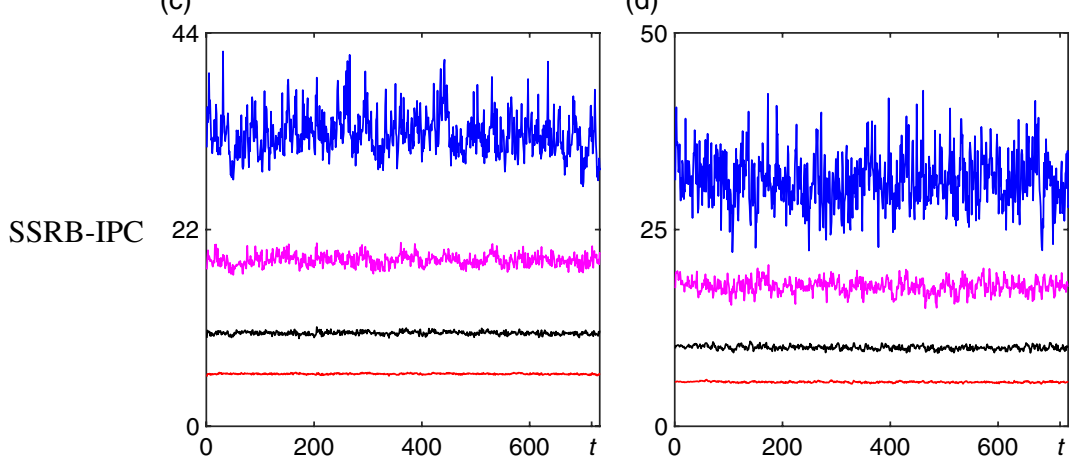

FIGURE 10. Examples of the infinitesimal standard deviation for attractiveness, $\sqrt{\mathscr{V}_{1}^{\ell,(q)}(t)}$, in the cases of zero hotspot formation and hotspot formation. For the SSRB model [104], (a) shows results in the cases with no hotspot. The blue, magenta, black and red lines show results with the simulations in Figures 1(a), (b), 2(a) and (b), respectively. (b) shows results with hotspot formation. The blue, magenta, black and red lines represent results with the simulations in Figs 3(a), 3(b), 4(a) and 4(b), respectively. For the SSRBIPC model, (c) shows results in the cases with no hotspot. The blue, magenta, black and red lines show results with the simulations in Figures 1(c), (d), 2(c) and (d), respectively. (d) shows results with hotspot formation. The blue, magenta, black and red lines represent results with the simulations in Figs 3(c), 3(d), 4(c) and 4(d), respectively.

blue, magenta, black and red lines represent results with the simulations in Figs 3(a), 3(b), 4(a) and 4(b), respectively.

For the agent-based SSRB-IPC model [104], Figure 10(c) shows results in the cases with no hotspot formation. The blue, magenta, black and red lines show results with the simulations in Figures 1(c), (d), 2(c) and (d), respectively. Figure 10(d) shows results with hotspot formation. The blue, magenta, black and red lines represent results with the simulations in Figs 3(c), 3(d), 4(c) and 4(d), respectively.

The output of the simulations supports the validity of (3.29). The same simulation results are also observed over other random paths.

Furthermore, we check with simulations the validity of (3.25) for an arbitrary later time $t>0$, which is

$$
\mathscr{V}_{1}^{\ell,(q)}\left(t, \phi^{\ell}\right) \cong \frac{1}{q} \ell^{2} \Theta^{2} \mathfrak{d}\left|\phi^{\ell}\right|_{2}^{2}, \quad t>0 .
$$


(a) $\log \left(\frac{\int_{T_{1}}^{T_{2}} \mathscr{V}_{1}^{\ell,(q)} d t}{T_{1}-T_{2}}\right)$

SSRB

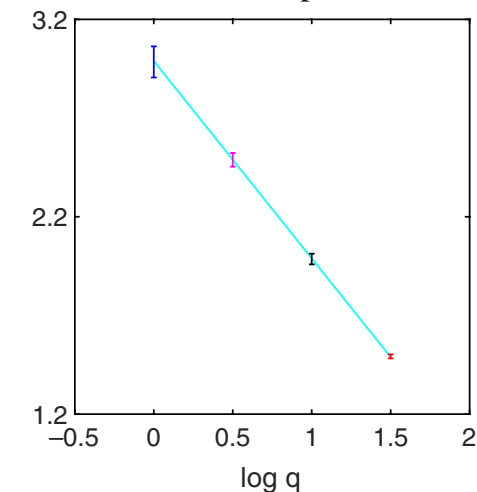

(c)

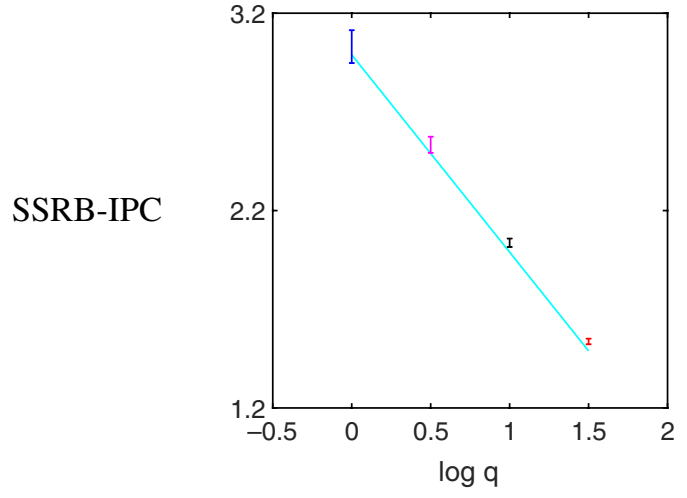

(b)

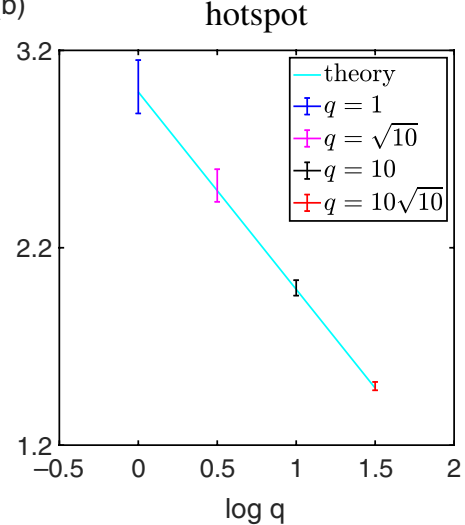

(d)

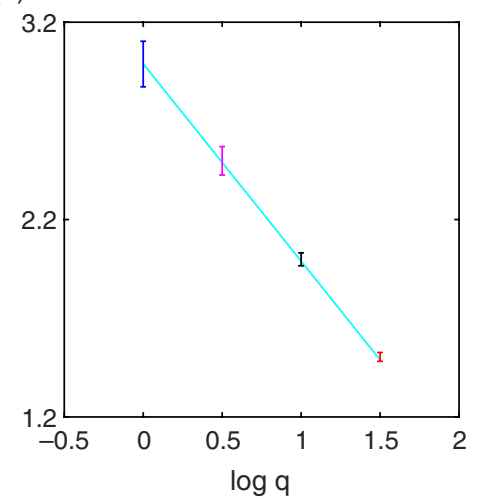

FIGURE 11. Comparison of the log-log plot of the theoretical and true scaling in (3.32) with error bars for both zero hotspot formation and hotspot formation. The straight lines show the theoretical scaling with slope -1 and the $x$-intercept $\ell^{2} \Theta^{2} \mathfrak{d}\left|\phi^{\ell}\right|_{2}^{2} \cong 2.9892$, where $\mathfrak{d}=0.019, \Theta=5.6$, and $\phi^{\ell}$ is assumed as in 3.30 . The error bars show the true scaling, with $\left[T_{1}, T_{2}\right]$ set as $[0,10],[1,11],[2,12], \ldots,[719,729],[720,730]$. For the SSRB model [104], (a) shows results with no hotspot, and the error bars with $x$-axis as $0,1,2$ and 3 show results with the simulations of the blue, magenta, black and red lines in Figure 10 (a), respectively. (b) shows results with hotspot formation, and the error bars with $x$-axis as $0,1,2$ and 3 show results with the simulations of the blue, magenta, black and red lines in Figure 10 (b), respectively. For the SSRB-IPC model, (c) shows results with no hotspot, and the error bars with $x$-axis as $0,1,2$, and 3 show results with the simulations of the blue, magenta, black and red lines in Figure 10 (c), respectively. (d) shows results with hotspot formation, and the error bars with $x$-axis as $0,1,2$ and 3 show results with the simulations of the blue, magenta, black and red lines in Figure 10 (d), respectively.

This is an analog of Equation (39) [104] for the SSRB model. In Figure 11, we run direct simulations for the SSRB model [104] and the SSRB-IPC model. Taking average of both sides of (3.31) over a time period $\left[T_{1}, T_{2}\right]$, we obtain

$$
\frac{1}{T_{2}-T_{1}} \int_{T_{1}}^{T_{2}} \mathscr{V}_{1}^{\ell,(q)}\left(t, \phi^{\ell}\right) d t \cong \frac{1}{q} \ell^{2} \Theta^{2} \mathfrak{d}\left|\phi^{\ell}\right|_{2}^{2} .
$$

Figure 11 shows the log-log plot with error bars for (3.32). The lines show the theoretical scaling with slope as -1 and the $x$-intercept as $\ell^{2} \Theta^{2} \mathfrak{d}\left|\phi^{\ell}\right|_{2}^{2}$, and the error bars show the true scaling with the $x$-coordinate and $y$-coordinate as: 


$$
x=\log q, \quad y=\log \left(\frac{1}{T_{2}-T_{1}} \int_{T_{1}}^{T_{2}} \mathscr{V}_{1}^{\ell,(q)}\left(t, \phi^{\ell}\right) d t\right), \quad q=1, \sqrt{10}, 10,10 \sqrt{10} .
$$

Here, $\left[T_{1}, T_{2}\right]$ is chosen as $[0,10],[1,11],[2,12], \ldots,[719,729],[720,730]$. The minimum and maximum values of $y$ taken over all such intervals are set as the limits of the error bars.

For the SSRB model [104], Figure 11 (a) shows results with no hotspot, and the error bars with $x$-axis as $0,1,2$ and 3 show results with the simulations of the blue, magenta, black and red lines in Figure 10 (a), respectively. Figure 11(b) shows results with hotspot formation, and the error bars with $x$-axis as $0,1,2$ and 3 show results with the simulations of the blue, magenta, black and red lines in Figure 10 (b), respectively.

For the SSRB-IPC model, Figure 11(c) shows results with no hotspot, and the error bars with $x$-axis as $0,1,2$ and 3 show results with the simulations of the blue, magenta, black and red lines in Figure 10 (c), respectively. Figure 11(d) shows results with hotspot formation, and the error bars with $x$-axis as $0,1,2$ and 3 show results with the simulations of the blue, magenta, black and red lines in Figure 10 (d), respectively.

The output shows that for the agent-based SSRB model [104] and the agent-based SSRBIPC model, the error bars are short and fall mostly on the straight lines representing the theory. Moreover, the larger the total number of burglars is, the shorter the error bar is. The same simulation results are also observed over other random paths. These results support the validity of (3.32) and our theory for the finite size effects based on the Martingale formulation.

\section{Conclusion}

In this article, independent Poisson clocks are applied to individual agents in the agent-based DTS model [93]. The time increments are independently exponentially distributed random variables, which are more suitable to model independent actions of agents. The agent-based SSRB-IPC model is more realistic than the agent-based SSRB model [104] where one Poisson clock governs all the agents. The SSRB and SSRB-IPC models are both models with stochastic features.

The simulations of the SSRB-IPC model produces aggregate pattern formation of residential burglary assuming independent agent actions. And the finite size effects are observed in the simulations: dynamic hotspots are observed associated with small burglar population.

The agent-based SSRB-IPC model is also an interacting particle system, and stochastic analysis is applicable. A Martingale formulation for the SSRB-IPC model is derived. The formula consists of a deterministic and a stochastic component, that is, the infinitesimal mean and the infinitesimal variance. The infinitesimal mean is very similar to that of the SSRB model (see (11) and (12) in [104]). And the infinitesimal mean yields a continuum equation, which coincides with the that of the DTS model [93] and of the SSRB model [104]. Simulations show that the continuum equation is a good approximation of the SSRB-IPC model under the circumstance of a large number of burglars. The infinitesimal variance of the SSRB-IPC model is less complicated than that of the SSRB model (see (13) and (14) in [104]). And we get more robust and rigorous results than [104].

Moreover, the pattern formation of crime hotspots is quantified by the statistics measuring the degree of transience of hotspots. A theory for the finite size effects is developed based on the Martingale formulation. As the burglar population decreases, the stochastic component of 
the Martingale representation increases in size, while the deterministic component remains the same, which leads to a larger stochastic fluctuation of the SSRB-IPC model from its continuum equation. The theory can be proven at time zero with equilibrium initial data, and we conjecture that it remains to be true at arbitrary later times with negligible error. Quantitative numerical simulations support our conjecture. The finite size effects are closely related to hotspot transience, which is well documented in real crime statistics. Therefore, through the observation of hotspot transience, we could estimate the number of criminals, something that is normally difficult to predict. For general human behaviour with similar aggregation pattern formation, our finding could help with the prediction of the size of agents participating in the activity.

\section{Acknowledgements}

We would like to thank the helpful discussions with Prof. A. Debussche, Prof. Thomas Liggett, Prof. Carl Mueller, Da Kuang, Yifan Chen, Wuchen Li, and Kenneth Van. A. Bertozzi was supported by NSF grant DMS-1737770 and M. Short was supported by NSF grant DMS-1737925.

\section{Conflict of interest}

None.

\section{References}

[1] Applebaum, D. (2009) Lévy Processes and Stochastic Calculus, Cambridge University Press, Cambridge.

[2] BABU, G. J. \& Feigelson, E. D. (2015) Spatial point processes in astronomy. J. Stat. Plan. Inference 50, 311-326.

[3] Bellomo, N., Colasuonno, F. \& Knopoff, D. \& Soler, J. (2015) From a systems theory of sociology to modeling the onset and evolution of criminality. Netw. Heterog. Media 10, 421-441.

[4] Berestycki, H., Rodrí, N. \& RyZhiK, L. (2013) Traveling wave solutions in a reaction-diffusion model for criminal activity. Multiscale Model. Simul. 11, 1097-1126.

[5] Bertero, M., Boccacci, P., Desiderà, G. \& Vicidomini, G. (2009) Image deblurring with Poisson data: from cells to galaxies. Inverse Prob. 25, 123006.

[6] Bertozzi, A. L., Johnson, S. \& WARD, M. J. (2016) Mathematical modeling of crime and security: special issue of EJAM, Euro. J. Appl. Math. 27, 311-316.

[7] Bertsekas, D. P. \& Tsitsiklis, J. N. (2008) Introduction to Probability, Athena Scientific, Belmont, Mass.

[8] Bichteler, K. (2002) Stochastic Integration with Jumps, Cambridge University Press, Cambridge.

[9] Brockmann, D., Hufnagel, L. \& And Geisel, T. (2006) The scaling laws of human travel. Nature 439, 462-465.

[10] BudD,T. (1999) Burglary of Domestic Dwellings: Findings from the British Crime Survey, Home Office Statistical Bulletin, Vol. , Government Statistical Service, London.

[11] Buttenschoen, A., Kolokolnikov, T., Ward, M. J. \& Wei, J. (2018) Cops-on-the-Dots: The linear stability of crime hotspots for a 1-D reaction-diffusion model of urban crime.

[12] Cantrell, R. And Cosner, C. And ManÁsevich, R. (2012) Global bifurcation of solutions for crime modeling equations. SIAM J. Math. Anal. 44, 1340-1358.

[13] CAO, L. \& GRABCHAK, M. (2014) Smoothly truncated levy walks: Toward a realistic mobility model. In: 2014 IEEE 33rd Int. Performance Computing and Communications Conference, (IPCCC), December 2014, Austin, Texas, USA, 1-8. 
[14] CHANG, Y., TZOU, J., WARD, M. \& WEI, J. (2018) Refined stability thresholds for localized spot patterns for the Brusselator model in $\mathbb{R}^{2}$. Euro. J. Appl. Math., 30, 791-828.

[15] Chaturapruek, S., Breslau, J., Yazdi, D., Kolokolnikov, T. \& McCalla, S. G. (2013) Crime modeling with Lévy flights. SIAM J. Appl. Math. 73, 1703-1720.

[16] CHOw, S. N., LI, W. \& ZHOU, H. (2018) Entropy dissipation of Fokker-Planck equations on graphs. Discrete Contin. Dyn. Syst. 38, 4929-4950.

[17] Chung, K. L. \& Williams, R. J. (2014) Introduction to Stochastic Integration, Birkhäuser/ Springer, New York.

[18] Citi, L., BA, D., Brown, E. N. \& BARbieri, R. (2014) Likelihood methods for point processes with refractoriness. Neural Comput. 26, 237-263.

[19] Ding, H., Khan, M. E., Sato, I. Grabchak, M. \& SugiYama, M. (2018) Bayesian nonparametric Poisson-process allocation for time-sequence modeling. In: Proceedings of the 21st International Conference on Artificial Intelligence and Statistics (AISTATS), Vol. 84, PMLR, Lanzarote, Spain.

[20] Durrett, R. (1996) Stochastic Calculus, CRC Press, Boca Raton, Florida.

[21] Durrett, R. (1999) Essentials of Stochastic Processes, Springer-Verlag, New York.

[22] Durrett, R. (2002) Probability Models for DNA Sequence Evolution, Springer-Verlag, New York.

[23] Durrett, R. (2010) Probability: Theory and Examples, Cambridge University Press, Cambridge.

[24] Ellam, L., Girolami, M., Pavliotis, G. A. \& Wilson, A. (2018) Stochastic modeling of urban structure. Proc. R. Soc. A. 474, pp. 1-20.

[25] Embrechts, P., Frey, R. \& FUrRer, H. (2001) Stochastic processes in insurance and finance. In: Stochastic Processes: Theory and Methods. North-Holland, Amsterdam, 365-412.

[26] Ethier, S. N. \& Kurtz, T. G. (1986) Markov Processes, John Wiley \& Sons, Inc., New York.

[27] ERBAR, M. \& MAAS, J. (2012) Ricci curvature of finite Markov chains via convexity of the entropy. Arch. Ration. Mech. Anal. 206, 997-1038.

[28] ERBAR, M. \& MAAS, J. (2014) Gradient flow structures for discrete porous medium equations. Discrete Contin. Dyn. Syst. 34, 1355-1374.

[29] FATHI, M. \& MAAS, J. (2016) Entropic Ricci curvature bounds for discrete interacting systems. Ann. Appl. Probab. 26, 1774-1806.

[30] Farrell, G. \& Pease, K. (2001) Repeat Victimization, Criminal Justice Press.

[31] FRANCO, T. (2014) Interacting particle systems: hydrodynamic limit versus high density limit. In: From Particle Systems to Partial Differential Equations, Springer, Heidelberg, pp. 179-189.

[32] GAU, J. M. \& PRATT, T. C. (2010) Revisiting broken windows theory: Examining the sources of the discriminant validity of perceived disorder and crime. J. Crim. Justice 38, 758-766.

[33] Gleeson, J. P., Hurd, T. R., Melnik, S. \& Hackett, A. (2018) Systemic risk in banking networks without Monte Carlo simulation, In: Advances in Network Analysis and its Applications. Springer, Berlin, pp. 27-56.

[34] Gleeson, J. P. \& Porter, M. A. (2018) Message-passing methods for complex contagions. In: Complex spreading phenomena in social systems. Computational Social Sciences. Springer, Cham.

[35] Gomez, D., WARD, M. J. \& WeI, J. (2019) The linear stability of symmetric spike patterns for a bulk-membrane coupled Gierer-Meinhardt model. SIAM J. Appl. Dyn. Sys. 18, 729-768.

[36] GonzÁlez, M. C., Hidalgo, C. A. \& BARABÁsi, A.-L. (2008) Understanding individual human mobility patterns. Nature $\mathbf{4 5 3}, 779-782$.

[37] Good, I. J. (1986) Some statistical applications of Poisson's work. Statist. Sci. 1, 157-180.

[38] GorR, W. \& LeE, Y. (2015) Early warning system for temporary crime hot spots. J. Quant. Criminol. 31, 25-47.

[39] Goudon, T., Nkonga, B., Rascle, M. \& Ribot, M. (2015) Self-organized populations interacting under pursuit-evasion dynamics. Phys. D 304/305, 1-22.

[40] Guo, M. Z., Papanicolaou, G. C. \& Varadhan, S. R. S. (1988) Nonlinear diffusion limit for a system with nearest neighbor interactions. Comm. Math. Phys. 118, 31-59.

[41] HambA, F. (2018) Turbulent energy density in scale space for inhomogeneous turbulence. J. Fluid Mech. 842, 532-553.

[42] Hameduddin, I., Meneveau, C., Zaki, T. A. \& Gayme, D. F. (2018) Geometric decomposition of the conformation tensor in viscoelastic turbulence. J. Fluid Mech. 842, 395-427. 
[43] Harcourt, B. E. (1998) Reflecting on the subject: A critique of the social influence conception of deterrence, the broken windows theory, and order-maintenance policing New York style. Michigan Law Rev. 97, 291-389.

[44] He, S. W., Wang, J. G. \& Yan, J. A. (1992) Semimartingale Theory and Stochastic Calculus, Kexue Chubanshe (Science Press), Beijing; CRC Press, Boca Raton, FL.

[45] Illingworth, S. J., Monty, J. P. \& MARUsic, I. (2018) Estimating large-scale structures in wall turbulence using linear models, J. Fluid Mech. 842, 146-162.

[46] JAcod, J. \& Shiryaev, A. N. (2003) Limit Theorems for Stochastic Processes, Springer-Verlag, Berlin.

[47] James, A., Plank, M. J. \& Edwards, A. M. (2011) Assessing Lévy walks as models of animal foraging. J. R. Soc. Interface 8, 1233-1247.

[48] JimÉnEZ, J. (2018) Coherent structures in wall-bounded turbulence. J. Fluid Mech. 842, P1.

[49] Johnson, S. D., Bernasco, W., Bowers, K. J., Elffers, H., Ratcliffe, J. Rengert, G., \& TOWNSLEY, M. (2007) Space-time patterns of risk: A cross national assessment of residential burglary victimization. J. Quant. Criminol. 23, 201-219.

[50] Johnson, S. D. \& Bowers, K. J. (2004) The stability of space-time clusters of burglary. Br. J. Criminol. 44, 55-65.

[51] Johnson, S. D., Bowers, K. J., \& Hirschfield, A. (1997) New insights into the spatial and temporal distribution of repeat victimization. Br. J. Criminol. 37, 224-241.

[52] Jones, P. A., BRAntingham, P. J. \& CHAYES, L. R. (2010) Statistical models of criminal behavior: the effects of law enforcement actions. Math. Models Methods Appl. Sci. 20, 1397-1423.

[53] Karlin, S. \& Taylor, H. M. (1981) A Second Course in Stochastic Processes, Academic Press, Inc. [Harcourt Brace Jovanovich, Publishers], New York-London.

[54] Kipnis, C. \& Landim, C. (1999) Scaling Limits of Interacting Particle Systems, Springer-Verlag, Berlin.

[55] Kipnis, C., Olla, S. \& Varadhan, S. R. S. (1989) Hydrodynamics and large deviation for simple exclusion processes. Comm. Pure Appl. Math. 42, 115-137.

[56] Kolokolnikov, T., WARD, M. J. \& Wei, J. (2014) The stability of steady-state hot-spot patterns for a reaction-diffusion model of urban crime. Discrete Contin. Dyn. Syst. Ser. B 19, 1373-1410.

[57] Kolokolnikov, T., Ward, M. J., Tzou, J. \& Wei, J. (2018) Stabilizing a homoclinic stripe. Philosophical Transactions of the Royal Society A: Mathematical, Physical and Engineering Sciences 376, 13 pages.

[58] Kolokolnikov, T. \& Wei, J. (2018) Pattern formation in a reaction-diffusion system with spacedependent feed rate. SIAM Review 60, 626-645.

[59] LACEY, A. \& TSARDAKAS, M. (2016) A mathematical model of serious and minor criminal activity. Euro. J. Appl. Math. 27, 403-421.

[60] Levajković, T., Mena, H. \& ZARfl, M. (2016) Lévy processes, subordinators and crime modeling. Novi. Sad. J. Math. 46, 65-86.

[61] LeE, C. \& Wilkinson, D. J. (2019) A Hierarchical model of nonhomogeneous Poisson processes for twitter retweets. J. Am. Stat. Assoc. 0, 1-23.

[62] Liggett, T. M. (1986) Lectures on stochastic flows and applications, Published for the Tata Institute of Fundamental Research, Bombay; Springer-Verlag, Berlin.

[63] Liggett, T. M. (1980) Interacting Markov processes. In: Biological Growth and Spread, Lecture Notes in Biomathematics, Vol. 38. Springer, Berlin, Heidelberg, pp. 145-156.

[64] Liggett, T. M. (1985) Interacting Particle Systems, Springer-Verlag, New York.

[65] Liggett, T. M. (2010) Continuous Time Markov Processes, American Mathematical Society, Providence, RI.

[66] Lloyd, D. J. B. \& O'Farrell, H. (2013) On localised hotspots of an urban crime model. Phys. D 253, 23-39.

[67] Lukasik, M., Cohn, T. \& Bontcheva, K. (2015) Point process modelling of rumour dynamics in social media. Proceedings of the 53rd Annual Meeting of the Association for Computational Linguistics and the 7th International Joint Conference on Natural Language Processing, (ACL), Beijing, China, July 2015, pp. 518-523. 
[68] Mantegna, R. N. \& Stanley, H. E. (1994) Stochastic process with ultraslow convergence to a Gaussian: the truncated Lévy flight. Phys. Rev. Lett. 73, 2946-2949.

[69] Mariani, M. C. \& LiU, Y. (2007) Normalized truncated Levy walks applied to the study of financial indices. Physica A: Stat. Mech. Appl. 377, 590-598.

[70] Ajmone Marsan, G., Bellomo, N. \& Gibelli, L. (2016) Stochastic evolutionary differential games toward a systems theory of behavioral social dynamics. Math. Models Methods Appl. Sci. 26, 1051-1093.

[71] Matacz, A. (2000) Financial modeling and option theory with the truncated Lévy process. Int. J. Theor. Appl. Financ. 3, 143-160.

[72] McCalla, S. G., Short, M. B. \& Brantingham, P. J. (2013) The effects of sacred value networks within an evolutionary, adversarial game. J. Stat. Phys. 151, 673-688.

[73] Métivier, M. (1982) Semimartingales, Walter de Gruyter \& Co., Berlin-New York.

[74] Métivier, M. \& Pellaumail, J. (1980) Stochastic Integration, Academic Press [Harcourt Brace Jovanovich, Publishers], New York-London-Toronto, Ont.

[75] Miranda L. C. \& RierA, R. (2001) Truncated Lévy walks and an emerging market economic index. Physica A: Stat. Mech. Appl. 297, 509-520.

[76] Mohler, G. O., Short, M. B. \& Brantingham, P. J. (2017) The concentration-dynamics tradeoff in crime hot spotting. In Unraveling the Crime-Place Connection, Vol. 22, Routledge, pp. 19-40.

[77] Mohler, G. O., Short, M. B. \& Brantingham, P. J., Schoenberg, F. P. \& Tita, G. E. (2011) Self-exciting point process modeling of crime. J. Am. Stat. Assoc. 106, 100-108.

[78] Mohler, G. O., Short, M. B., Malinowski, S., Johnson, M., Tita, G. E., Bertozzi, A. L. \& Brantingham, P. J. (2015) Randomized controlled field trials of predictive policing, J. Am. Stat. Assoc., 110, 1399-1411.

[79] Mourrat, J. (2012) A quantitative central limit theorem for the random walk among random conductance, Electron. J. Probab. 17, 17.

[80] Othmer, H. G., Dunbar, S. R. \& Alt, W. (1988) Models of dispersal in biological systems. J. Math. Biol. 26, 263-298.

[81] Pan, C., Li, B., Wang, C., Zhang, Y., Geldner, N., Wang, L. \& Bertozzi, A. L. (2018) Crime modeling with truncated Lévy flights for residential burglary models. Math. Models Methods Appl. Sci. 28, 1857-1880.

[82] Paquin-Lefebvre, F., Nagata, W. \& Ward, M. J. (2019) Pattern formation and oscillatory dynamics in a 2-D coupled bulk-surface reaction-diffusion system. SIAM J. Appl. Dyn. Syst.18, 1334-1390.

[83] Peszat, S. \& ZABczyK, J. (2007) Stochastic partial differential equations with Lévy noise, Cambridge University Press, Cambridge.

[84] Pitcher, A. B. (2010) Adding police to a mathematical model of burglary. Euro. J. Appl. Math. 21, 401-419.

[85] Protter, P. E. (2005) Stochastic Integration and Differential Equations, Springer-Verlag, Berlin.

[86] RodRíGUEZ, N. (2013) On the global well-posedness theory for a class of PDE models for criminal activity. Phys. D 260, 191-200.

[87] Rodríguez, N. \& BertozzI, A. L. (2010) Local existence and uniqueness of solutions to a PDE model for criminal behavior. Math. Models Methods Appl. Sci. 20, 1425-1457.

[88] Saldaña, J., Aguareles, M., Avinyó, A., Pellicer, M. \& Ripoll, J. (2018) An age-structured population approach for the mathematical modeling of urban burglaries. SIAM J. Appl. Dyn. Syst. 17, 2733-2760.

[89] Shen, H., ANd Wang, D., Song, C. \& BARABÁsi, A. (2014) Modeling and predicting popularity dynamics via reinforced poisson processes. In: Proceedings of the Twenty-Eighth AAAI Conference on Artificial Intelligence, AAAI Press, pp. 291-297.

[90] Short, M. B., Bertozzi, A. L. \& Brantingham, P. J. (2010) Nonlinear patterns in urban crime: hotspots, bifurcations, and suppression. SIAM J. Appl. Dyn. Syst. 9, 462-483.

[91] Short, M. B., Brantingham, P. J., Bertozzi, A. L. \& Tita, G. E. (2010) Dissipation and displacement of hotspots in reaction-diffusion models of crime. Proc. Natl. Acad. Sci. 107, 39613965. 
[92] Short, M. B., D’Orsogna, M. R., Brantingham, P. J. \& Tita, G. E. (2009) Measuring and modeling repeat and near-repeat burglary effects. J. Quant. Criminol. 25, 325-339.

[93] Short, M. B., D’Orsogna, M. R., Pasour, V. B., Tita, G. E., Brantingham, P. J., Bertozzi, A. L. \& Chayes, L. B. (2008) A statistical model of criminal behavior. Math. Models Methods Appl. Sci. 18, 1249-1267.

[94] Short, M. B., Mohler, G. O., Brantingham, P. J. \& Tita, G. E (2014) Gang rivalry dynamics via coupled point process networks. Discrete Contin. Dyn. Syst. Ser. B 19, 1459-1477.

[95] B. SNOOK (2004) Individual differences in distance travelled by serial burglars. J. Investig. Psych. Offender Profil. 1, 53-66.

[96] Stroock, D. W. \& Varadhan, S. R. S. (2006) Multidimensional Diffusion Processes. SpringerVerlag, Berlin.

[97] То́тн, B. \& VALKó, B. (2003) Onsager relations and Eulerian hydrodynamic limit for systems with several conservation laws. J. Statist. Phys. 112, 497-521.

[98] TSE, W. H. \& WARD, M. J. (2016) Hotspot formation and dynamics for a continuum model of urban crime. Euro. J. Appl. Math. 27, 583-624.

[99] TSE, W. H., \& WARD, M. J. (2018). Asynchronous instabilities of crime hotspots for a 1-D reactiondiffusion model of urban crime with focused police patrol. SIAM J. Appl. Dyn. Syst. 17, 2018-2075.

[100] Tzou, J., WARD, M. J \& WEI, J. (2018) Anomalous scaling of Hopf bifurcation thresholds for the stability of localized spot patterns for reaction-diffusion systems in 2-D. SIAM J. Appl. Dyn. Sys. 17, 982-1022.

[101] VAn Koppen, P. J. \& JAnsen, R. W. J. (1998) The road to the robbery: Travel patterns in commercial robberies. Brit. J. Criminol. 38, 230-246.

[102] VARAdhan, S. R. S. (1994) Entropy methods in hydrodynamic scaling. In: Proceedings of the International Congress of Mathematicians, Vol. 1, 2, Zürich, Birkhäuser, Basel, pp. 196-208,

[103] Varadhan, S. R. S. (2000) Lectures on hydrodynamic scaling. In: Hydrodynamic Limits and Related topics, Amer. Math. Soc., Providence, RI, pp. 3-40.

[104] WANG, C., ZhAng, Y., BertozZI , A. L. \& ShORT, M. B (2019) A stochastic-statistical residential burglary model with finite size effects. In: Active Particles., Vol. 2, Modeling and Simulation in Science, Engineering and Technology, Springer Nature Switzerland AG, in press.

[105] WARD, M. J. (2018) Spots, traps, and patches: asymptotic analysis of localized solutions to some linear and nonlinear diffusive processes. Nonlinearity 31, R189 (53 pages).

[106] Wilso, J. Q. \& Kelling, G. L. (1982) Broken windows: The police and neighborhood safety, Atlantic Mon. 249, 29-38.

[107] Wu, Y., Zhou, C., Xiao, J., Kurths, J. \& Schellnhuber, H. J. (2010) Evidence for a bimodal distribution in human communication. Proc. Natl. Acad. Sci. U S A. 105, 18803-18808.

[108] Zipkin, J. R., Short, M. B. \& Bertozzi, A. L. (2014) Cops on the dots in a mathematical model of urban crime and police response. Discrete Contin. Dyn. Syst. Ser. B 19, 1479-1506. 